wsp

\title{
ON QUANTIFICATION WITH A FINITE UNIVERSE
}

\author{
SAHARON SHELAH \\ Institute of Mathematics \\ The Hebrew University \\ Jerusalem, Israel \\ Rutgers University \\ Mathematics Department \\ New Brunswick, NJ USA
}

\begin{abstract}
We consider a finite universe $\mathcal{U}$ (more exactly - a family $\mathfrak{U}$ of them), second order quantifiers $Q_{K}$, where for each $\mathcal{U}$ this means quantifying over a family of $n(K)$-place relations closed under permuting $\mathcal{U}$. We define some natural orders and shed some light on the classification problem of those quantifiers.
\end{abstract}

I would like to thank Alice Leonhardt for the beautiful typing.

Publication No. 639

First Typed - 97/Dec/19

Latest Revision - 98/Oct/27 


\section{Annotated Content}

Introduction

[We explain our problem: classifying second order quantifiers for finite model theory. We review relevant works, mainly, the work done on infinite ones. We then define the basic order relations on such quantifiers interpretability and expressability. We also explain why they are reasonable: as for definable quantifiers, those give the desired recursiveness result.]

On some specific quantifiers

[We define the quantifier we shall use: monadic, partial one-to-one functions, equivalence relations and linear order. All have versions with a cardinality restriction (say cardinality of the domain of a one-to-one function), which depends on $\mathscr{U}$, the universe, only. For example $Q_{\leq \lambda}^{\text {mon }}$ is the quantifier over sets of cardinality $\leq \lambda, Q_{\leq \lambda}^{1-1}$ is the quantifier over unary one-to-one functions with domain of cardinality $\leq \lambda$, and $Q^{\text {eq }}$ the quantifier over equivalence relations. We shall investigate the natural partial orders on them (by the so-called interpretability and expressibility).]

Monadic analyses of $\exists_{R}$

[Concentrating first on $\exists_{R}$, quantifying on the isomorphic copies of one $n(R)$-place relation $R$, we try to analyze its "monadic content". We essentially characterize the maximal cardinality of a set interpreted by cases of $R$ by a first order formula (actually of low quantifier depth) as $\lambda_{0}(R)$ and show that using such a set we can reduce $R$ to $R_{1}$ which has domain of cardinality $\lambda_{0}(R)$. So up to bi-interpretability, $Q_{R}$ and $\left\{Q_{R_{1}}, Q_{\leq \lambda_{0}(R)}\right\}$ are equivalent. Now when $\lambda_{0}(R)$ is too near to the cardinality of the universe $\mathscr{U}$, we have to be more careful but we interpret the (full) monadic quantifier (with no cardinality restriction). Lastly, we do the same for $Q_{K}$.]

The one-to-one function analysis

[We define a cardinal $\lambda_{1}(R)$ which essentially characterizes the maximal cardinality of the domain of a one-to-one function interpretable by cases of $R$. It is called $\lambda_{1}(R)$ and we can find a set $A \subseteq \mathscr{U}$ such that the order of magnitude of its cardinality is $\lambda_{1}(R)$ (here - a constant multiple), and show that $Q_{R}$ is equivalent by bi-interpretability to $\left\{Q_{R_{1}}, Q_{E}^{\mathrm{eq}}\right\}$ where $E$ is an equivalence relation with not too many equivalence classes and $R_{1}$ has domain of cardinality $\sim \lambda_{1}(R)$. Of course, $Q_{K}$ is analyzed similarly. Now, unlike the infinite case, up to bi-expressability $Q_{\lambda}^{1-1}$ is maximal in the sense that if $R_{1}$ has domain $\leq \lambda^{1 / n(R)}$ then it is expressible by $Q_{\lambda}^{1-1}$. Hence, under bi-expressibility and up to polynomial order of magnitude we have a complete classification. Of course, on top of $Q_{\lambda_{1}(R)}^{1-1}$ we have the equivalence relation, which is understood.] 


\section{$\S 0$ INTRODUCTION}

We investigate and classify to a large extent quantifiers in the following framework

(*) for a natural number $n$, for a (large) finite set $\mathscr{U}$, consider a quantifier $Q_{K}$ on $n$-place relations on $\mathscr{U}$, so $K$ is a family of $n$-place relations on $\mathscr{U}$ close under isomorphism (i.e. permutation of $\mathscr{U}$ ).

It is natural to restrict ourselves to such families defined by the logic we have in mind (usually first order), but it seems natural to investigate two partial orders, interpretability and expressibility defined below, which for such definable classes give the right answer so the use of definability occurs only in the conclusion.

Earlier this was investigated for infinite $\mathscr{U}$, see (below and) in [Sh 28], [Bl], [Sh 171], but though related, there are some differences. A related work is [BlSh 156] which deals mainly with monadic logic on the class of models of a first order theory $T$, so its complicatedness measures the complexity of $T$. We have said on some occasion during this decade that those are adaptable to finite model theory. Here we deal with this and shall continue in [Sh:F334].

In [Sh 28] we gave a complete classification of the class of second order quantifiers: those which are first-order definable (see below an exact definition). We find that for infinite models up to a very strong notion of equivalence, bi-interpretability, there are only four such quantifiers: first order, monadic, one-to-one partial functions, and second-order. See Baldwin [Bl].

Now $\S 1-\S 3$ of the present work are parallel to $\S 1, \S 2, \S 3$ of [Sh 171], so below we describe the latter and then explain what we shall do here. In [Sh 171] our aim was to see what occurs if we remove the restriction that the quantifier is first-order definable. As we do not want to replace this by a specific $\mathscr{L}$-definable $(\mathscr{L}$-some logic) we restricted ourselves in [Sh 171] to a fixed infinite universe $\mathscr{U}$. If we then want to restrict ourselves to $\mathscr{L}$-definable quantifiers, we are able to remove the restriction to a fixed universe $\mathscr{U}$.

The strategy in [Sh 171] is to squeeze the quantifier $Q_{R}$ (similarly for $Q_{K}$ ) between some well understood quantifiers to get, eventually, equality. Unfortunately, for interpretability we get a lower bound and an upper bound which are close but not necessarily equal; i.e. both of the form $Q_{\mathbf{E}}$, where $\mathbf{E}$ is a set of equivalence relations and they are quite close (see below). More specifically we use cases of $Q_{\lambda, \mu}^{\mathrm{eq}}$ (i.e. on equivalence relations with $\lambda$ classes each of cardinality $\leq \mu$ ). Carrying out the strategy we first "find" the monadic content of, say, $Q_{R}$, by interpreting in it $Q_{\lambda_{0}(R)}^{\text {mon }}$ which is quantifying on sets of cardinality $\leq \lambda_{0}(R)$ and $\lambda_{0}(R)$ is maximal (and reduce the problem to "the remainder", that is a relation $R_{1}$ with $\operatorname{Dom}\left(R_{1}\right)$ of cardinality $\leq \lambda_{0}(R)$ and $\left.Q_{R_{1}} \leq_{\text {int }} Q_{R}\right)$. Next interpret $Q_{\lambda_{1}(R)}^{1-1}$ which is quantifying on partial one-to-one functions of cardinality $\leq \lambda_{1}(R)$. Now we succeed to squeeze $Q_{R}$, for "the remainder" between $Q_{\lambda, \lambda}$ and $Q_{\mu, \mu}, \lambda \leq \mu \leq \operatorname{Min}\left\{2^{\lambda},|\mathscr{U}|\right\}$ but in general cannot show this with $\lambda=\mu$. Clearly if $|\mathscr{U}|$ is $\aleph_{0}$, this does not occur and we can get a complete picture (see below 1.2). Also by "expressibility" (a stronger equivalence relation but O.K. for the application to logic) if $V=L$, then the gap does not occur, but in some generic extensions it does.

So by [Sh 171] we can e.g. conclude 
1.1 Theorem. Assume $K$ is a family of n-place relations over $\mathscr{U}$ where $|\mathscr{U}|=\aleph_{0}$. $\underline{\text { Then }} Q_{K}$ is bi-interpretable (see below) with $Q_{\mathbf{E}}$ for some family $\mathbf{E}$ of equivalence relations.

We can make this more specific.

The present situation is more complicated. For example, the finite cardinalities allow a family of monadic quantifiers: for the case $|\mathscr{U}|=n$ we have $Q_{\ln n}, Q_{\ln \ln \text { n }}$, etc. However, modulo these cardinality restrictions we are able to get a picture analogous to the original case. Also in the fine analysis we do not get an equivalence relation $E$ on $\mathscr{U}$ such that $Q_{R}, Q_{E}$ are bi-interpretable or even just bi-expressible, but just "squeeze" $Q_{R}$ between two such quantifiers, which are quite closed (i.e. size of one bounded by polynomial in the size of another). That is (concentrating on the case $\mathscr{U}$ is fixed (and finite)): assume $R$ is an $n$-place relation on $\mathscr{U}$ then we can uniformly attach it to a cardinal $\lambda_{1}(R)$, and an equivalence relation $E$ such that:

( $\alpha) Q_{E}^{\mathrm{eq}}, Q_{\lambda_{1}(R)}^{1-1}$ are interpretable in $Q_{R}$ (quantifiers over equivalence relations isomorphic to $E$ and partial 1 - 1-functions of cardinality $\left.\leq \lambda_{1}(R)\right)$

( $\beta)$ if $\lambda=\lambda_{1}(R)^{n(R)} \leq|\mathscr{U}| \underline{\text { then }} Q_{R}$ is expressible by $\left(Q_{E}^{\mathrm{eq}}, Q_{\lambda}^{1-1}\right)$

$(\gamma)$ if $\lambda_{1}(R)^{n(R)}>|\mathscr{U}|$, then any binary relation on a set $A \subseteq U$ with cardinality $|A|^{\frac{1}{2 n(R)}}$ is interpretable in $Q_{R}$.

The uniformly means that the formulas involved in interpretability or expressibility does not depend on $R$ and $\mathscr{U}$ but on $n$, in fact we can give explicit bounds on their size from $n$.

Note that we abuse notation using $R$ as a relation and predicate; of course, the formulas have an $n$-place predicate to stand for copies of $R$ (see below).

Note we actually deal also with quantifying on appropriate families of $R$ 's of fix arity (e.g. those satisfying some sentence). Note that we cannot get much better results by counting.

We thank C. Steinhorn, J. Tyszkiewicz and J. Baldwin for helpful discussions on preliminary versions in MSRI 10/89, Dimacs 95/96 and Rutgers Fall 1997, respectively. Much more is due to Baldwin, Fall 1998, for helping to greatly improve the presentation.

Let us now make some conventions and definitions.

1.2 Convention. 1) Informally $\mathscr{U}$ will be a fixed finite universe (usually large compared to $n$ ) but, if not said otherwise, we are proving things uniformly. So more exactly, $\mathscr{U}$ varies on $\mathfrak{U}$, a family of such sets. You may choose $\mathfrak{U}=\{(0, n)$ : $n$ a natural number $\}$.

2 ) Informally, $K$ will denote a family of $n$-place relations over $\mathscr{U}$, (for a natural number $n=n(K)$ ), closed under isomorphism, i.e. if $R_{1}, R_{2}$ are $n$-place relations on $\mathscr{U}$ and $\left(\mathscr{U}, R_{1}\right) \cong\left(\mathscr{U}, R_{2}\right)$ then $R_{1} \in K$ iff $R_{2} \in K$. So formally $K$ is a function with domain $\mathfrak{U}$ and $K[\mathscr{U}]$ is as above; but $n(K)=n(K[\mathscr{U}])$ for each $\mathscr{U} \in \mathfrak{U}$. Also 
below without saying in e.g. Definition 1.5 the formula $\varphi$ is the same for all $\mathscr{U} \in \mathfrak{U}$. 3) Let $\bar{K}$ denote a finite sequence of such $K$ 's, that is

$$
\bar{K}=\left\langle K_{\ell}: \ell<\ell g(\bar{K})\right\rangle \text {, so } \bar{K}_{i}^{j}=\left\langle K_{i, \ell}^{j}: \ell<\ell g\left(\bar{K}_{i}^{j}\right)\right\rangle \text {. }
$$

4) Let $R$ denote a relation, its domain is $\operatorname{Dom}(R)=\cup\{\bar{a}: \mid=R(\bar{a})\}, n=n(R)$ if $R$ is an $n$-place relation (or predicate; we shall not always strictly distinguish). Usually $R$ is on $\mathscr{U}$ which is clear from the context. Formally, $R$ is a function with domain $\mathfrak{U}$ and $R[\mathscr{U}]$ is an $n(R)$-place relation on $\mathscr{U})$.

1.3 Definition. For any $K, \exists_{K}$ (or $Q_{K}$ ) denotes a second order quantifier, intended to vary on members of $K$. More exactly, $L\left(\exists_{K_{1}}, \ldots, \exists_{K_{m}}\right)$ is defined like first order logic but we have for each $\ell=1, m$ (infinitely many) variables $R$ which serve as $n\left(K_{\ell}\right)$-place predicates, and we can form $\left(\exists_{K_{i}} R\right) \varphi$ for a formula $\varphi$ (when $R$ is $n\left(K_{i}\right)$-place). Defining satisfaction, we look only at models with universe $\mathscr{U}$, and $=\left(\exists_{K_{\ell}} R\right) \varphi(R, \cdots)$ iff for some $R^{0} \in K_{\ell}[\mathscr{U}]$ we have $\varphi\left(R^{0}, \cdots\right)$.

We may display the predicates (or relations) appearing in $\varphi$, i.e. $\varphi(x, y, \bar{R})$. Of course, we may write $K$ not $K[\mathscr{U}]$, etc., abusing notation.

Remark. Note that quantifiers depending on parameters are not allowed, e.g. automorphisms; on such quantifiers see [Sh:e].

1.4 Definition. We say that $K$ (or $Q_{K}$ ) is $\mathscr{L}$-definable (where $\mathscr{L}$ is a logic) if there is a formula $\varphi(R) \in \mathscr{L}$, in the vocabulary $\{R\}$ and is appropriate, i.e. an $n(K)$-place predicate, such that for any $n$-place relation $R$ on $\mathscr{U}$

$$
(\mathscr{U}, R) \models \varphi(R) \text { iff } R \in K \text {. }
$$

1.5 Definition. 1) We say that $\exists_{K_{1}} \leq_{\text {int }} \exists_{K_{2}}$ (in other words $\exists_{K_{1}}$ is interpretable in $\exists_{K_{2}}$ ) if for some first-order formula $\varphi(\bar{x}, \bar{S})=\varphi\left(x_{0}, \ldots, x_{n\left(K_{1}\right)-1}, S_{0}, \ldots, S_{m-1}\right)$, (each $S_{\ell}$ is an $n\left(K_{2}\right)$-place predicate) the following holds:

(*) for every $\mathscr{U} \in \mathfrak{U}$ and $R_{1} \in K_{1}[\mathscr{U}]$ there are $S_{0}, \ldots, S_{m-1} \in K_{2}[\mathscr{U}]$ such that $\left(\mathscr{U}, S_{0}, \ldots, S_{m-1}\right) \models(\forall \bar{x})\left[R_{1}(\bar{x}) \equiv \varphi\left(\bar{x}, S_{0}, \ldots, S_{m-1}\right)\right]$

(so in $(*), \varphi$ does not depend on $\mathscr{U}$ ).

2) We say $k$-interpretable if we demand $m \leq k$, and then write $\leq_{k \text {-int }}$.

3) We can define $\exists_{K_{1}} \leq \leq_{\text {int }}^{\mathscr{L}} \exists_{K_{2}}$ or $\exists_{K_{1}} \leq_{\text {int }} \exists_{K_{2}} \bmod \mathscr{L}$ similarly, by letting $\varphi \in \mathscr{L}$.

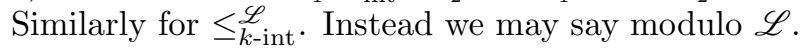

We define a weaker relative of interpretability; we say $\exists_{K_{1}}$ is expressible by $\exists_{K_{2}}$ if in the notion of interpretable we take the formula $\varphi$ to be in the logic $L\left(\exists_{K_{2}}\right)$. This is then a special but very important case of 1.5(3). 
1.6 Definition. 1) We say that $\exists_{K_{1}} \leq$ exp $\exists_{K_{2}}$ (in other words $\exists_{K_{1}}$ is expressible by $\left.\exists_{K_{2}}\right)$ if there is a formula $\varphi\left(\bar{x}, S_{0}, \ldots, S_{m-1}\right)$ in the logic $L\left(\exists_{K_{2}}\right)$ such that:

(*) for every $\mathscr{U} \in \mathfrak{U}$ and $R_{1} \in K_{1}[\mathscr{U}]$, there are $S_{0}, \ldots, S_{m-1} \in K_{2}[\mathscr{U}]$ such that $\left(\mathscr{U}, S_{0}, \ldots, S_{m-1}\right) \models(\forall \bar{x})\left[R_{1}(\bar{x}) \equiv \varphi\left(\bar{x}, S_{0}, \ldots, S_{m-1}\right)\right]$.

2) We say that $\exists_{K_{1}} \leq_{\text {inex }} \exists_{K_{2}}$ (in other words $\exists_{K_{2}}$ is invariantly expressible by $\left.\exists_{K_{2}}\right)$ if there is a formula $\varphi\left(\bar{x}, S_{0}, \ldots, S_{m-1}\right)$ in the logic $L\left(\exists_{K_{2}}\right)$ such that:

(*) for every $\mathscr{U} \in \mathfrak{U}$ and $R_{1} \in K_{1}[\mathscr{U}]$, there are $S_{0}, \ldots, S_{m-1} \in K_{2}[\mathscr{U}]$ such that for every $K_{3}$ which extends $K_{2}$, letting $\varphi^{\prime}$ be $\varphi$ when we replace $\exists_{K_{2}}$ by $\exists_{K_{3}}$ we have:

$$
\left(\mathscr{U}, S_{0}, \ldots, S_{m-1}\right) \models(\forall \bar{x})\left[R_{1}(\bar{x}) \equiv \varphi^{\prime}\left(\bar{x}, S_{0}, \ldots, S_{m-1}\right)\right] .
$$

3) We define $k$-expressible, $\leq_{k \text {-exp }}$, invariantly $k$-expressible and $\leq_{k \text {-inex }}$ and may add $\mathscr{L}$ as a superscript parallel to $1.5(2)$.

1.7 Definition. 1) We say that $\exists_{K_{1}} \equiv_{\text {int }} \exists_{K_{2}}$ (in other words $\exists_{K_{1}}, \exists_{K_{2}}$ are biinterpretable) if $\exists_{K_{1}} \leq_{\text {int }} \exists_{K_{2}}$ and $\exists_{K_{2}} \leq$ int $\exists_{K_{1}}$.

2) We say $\exists_{K_{2}} \equiv \equiv_{\exp } \exists_{K_{2}}$ (in other words $\exists_{K_{1}}, \exists_{K_{2}}$ are bi-expressible) if $\exists_{K_{1}} \leq$ exp $\exists_{K_{2}}$ and $\exists_{K_{2}} \leq \exp \exists_{K_{1}}$. Similarly for $\equiv_{\text {inex }}: \exists_{K_{1}} \equiv_{\text {inex }} \exists_{K_{2}}$ (in other words $\exists_{K_{2}}, \exists_{K_{1}}$ are invariantly bi-expressible) if $\exists_{K_{1}} \leq_{\text {inex }} \exists_{K_{2}}$ and $\exists_{K_{2}} \leq_{\text {inex }} \exists_{K_{1}}$.

3) We can define $\exists_{K_{1}} \leq$ int $\left\{\exists_{K_{0}}, \ldots, \exists_{K_{k-1}}\right\}$ as in Definition 1.5 but $S_{0}, \ldots, \in$ $\bigcup_{i=1}^{k} K_{i}[\mathscr{U}]$, we let $\exists_{\bar{K}}$ stand for $\left\{\exists_{K_{0}}, \ldots, \exists_{K_{k-1}}\right\}$ where $K=\left\langle K_{0}, \ldots, K_{k-1}\right\rangle$; we define $\exists_{\bar{K}^{1}} \leq_{\text {int }} \exists_{\bar{K}^{2}}$ if $\exists_{K_{\ell}^{1}} \leq_{\text {int }} \exists_{\bar{K}^{2}}$ for each $\ell$; we also define expressible, invariantly expressible, bi-interpretable and (invariantly) bi-expressible similarly.

4) Let $\exists_{K_{1}} \equiv_{1 \text {-int }} \exists_{K_{2}}$ (in other words $\exists_{K_{1}}, \exists_{K_{2}}$ are 1-bi-interpretable) if $\exists_{K_{1}} \leq_{1 \text {-int }}$ $\exists_{K_{2}}$ and $\exists_{K_{2}} \leq_{1 \text {-int }} \exists_{K_{1}}$; recall $\leq_{1 \text {-int }}$ is defined in $1.5(2)$ for $k=1$. Similarly $\exists_{K_{1}} \equiv_{1 \text {-exp }} \exists_{K_{2}}$ and $\exists_{K_{1}} \equiv_{1 \text {-inex }} \exists_{K_{2}}$.

5) In all those notions we add "modulo $\bar{K}$ " if parameters from $\cup\left\{K_{\ell}: \ell<\ell g(\bar{K})\right\}$ are allowed. We can combine this with $1.5(3)$ so have modulo $(\bar{K}, \mathscr{L})$.

1.8 Notation. 1) If $R_{\ell}$ is an $n_{\ell}$-place relation for $\ell<n$ then we let $\sum_{\ell=0}^{n-1} R_{\ell}=$ $\left\{\bar{a}_{0}{ }^{\wedge}{ }^{\wedge} \bar{a}_{n-1}: \bar{a}_{\ell} \in R_{\ell}\right\} ;$ more formally $\left(\sum_{\ell=0}^{n-1} R_{\ell}\right)(\mathscr{U})=\sum_{\ell=0}^{n-1} R_{\ell}[\mathscr{U}]$.

2) Let $\sum_{\ell=0}^{n-1} K_{\ell}=\left\{\sum_{\ell=0}^{n-1} R_{\ell}: R_{\ell} \in K_{\ell}\right.$ for $\left.\ell<n\right\}$.

3) $\exists_{R}$ stands for $\exists_{K}$ where $K=\left\{R_{1}:\left(\mathscr{U}, R_{1}\right) \cong(\mathscr{U}, R)\right\}$ and so formally if $R=\langle R[\mathscr{U}]: \mathscr{U} \in \mathfrak{U}\rangle$ then $K_{R}$ is defined by $K_{R}[\mathscr{U}]=\left\{R_{1}:\left(\mathscr{U}, R_{1}\right) \cong(\mathscr{U}, R)\right\}$. 
1.9 Lemma. 1) $\leq_{\text {int }}, \leq_{\text {inex }}$ and $\leq_{\exp }$ as well as $\leq_{1 \text {-int }}, \leq_{1 \text {-inex }}$ and $\leq_{1 \text {-exp }}$ are partial quasi orders. Hence $\equiv_{\mathrm{int}}, \equiv_{\mathrm{inex}}, \equiv_{\exp }$ are equivalence relations as well as $\equiv_{1 \text {-int }}$ , $\equiv_{1 \text {-inex }}$ and $\equiv_{1 \text {-exp }}$.

2) $\exists_{\bar{K}_{1}} \leq_{\text {int }} \exists_{\bar{K}_{2}}$ implies $\exists_{\bar{K}_{1}} \leq_{\text {inex }} \exists_{\bar{K}_{2}}$ which implies $\exists_{\bar{K}_{1}} \leq_{\exp } \exists_{\bar{K}_{2}}$. Similarly for the "1-" versions. Also each "1-" version implies the one without.

3) $\exists_{\bar{K}}$ and $\exists_{K}$ are bi-interpretable if $K=\sum_{i} K_{i}$ or $K=\bigcup_{i} K_{i}$ (where $n\left(K_{i}\right)$ constant in the second case).

4) In all those cases we can do everything modulo $\bar{K}_{0}$ or modulo $\mathscr{L}$ (if $\mathscr{L}$ is a reasonable logic closed by first order operations) or modulo $\left(\bar{K}_{0}, \mathscr{L}\right)$.

Proof. Straight.

1.10 Lemma. 1) If $\bar{K}_{1}, \bar{K}_{2}$ are $\mathscr{L}$-definable (i.e. each $K_{\ell, i}$ is, see Definition 1.4) and $\exists_{\bar{K}_{1}} \leq \exp \exists_{\bar{K}_{2}}$ then we can recursively attach to every formula in $\mathscr{L}\left(\exists_{\bar{K}_{1}}\right)$ an equivalent formula in $\mathscr{L}\left(\exists_{\bar{K}_{2}}\right)$.

2) If $\bar{K}_{1}, \bar{K}_{2}$ are $\mathscr{L}$-definable, $\exists_{\bar{K}_{1}} \leq{ }_{\exp } \exists_{\bar{K}_{2}}$ then the set of valid $\mathscr{L}\left(\exists_{\bar{K}_{1}}\right)$-sentences that is $\mathscr{L}\left(\exists_{K_{1,0}}, \ldots, \exists_{K_{1, \ell g(\bar{K})-1}}\right)$-sentences, recursive in the set of valid $\mathscr{L}\left(\exists_{\bar{K}_{2}}\right)$ sentences.

Proof. Easy.

Remark. 1) The need of " $\mathscr{L}$-definable" is clearly necessary. Though at first glance the conclusions of 1.10 may seem the natural definition of interpretable, I think reflection will lead us to see it isn't.

2) Note that naturally we use 1.10 with 1.9 .

3) Note that, of course, in 1.10, it is understood that the formulas from $\mathscr{L}$ are the same for all $\mathscr{U} \in \mathfrak{U}$. 


\section{$\S 1$ ON SOME SPECIFIC QUANTIFIERS}

2.1 Definition. 0) $K^{\text {tr }}=\{A \subseteq \mathscr{U}:|A|=1\}$, and we can write $\exists$ for $\exists_{K^{\text {tr }}}$; here tr stands for trivial.

1) Let $K_{\lambda}^{\text {mon }}=\{A \subseteq \mathscr{U}:|A|=\lambda\}$ for a number $\lambda \leq|\mathscr{U}| / 2$; here mon stands for monadic.

2) But we write $Q_{\lambda}^{\text {mon }}$ for $\exists_{K_{\lambda}^{\text {mon }}}$, and similarly for the other quantifiers defined below.

3) $K_{\lambda}^{1-1}=\{f: f$ is a partial one-to-one function, $|\operatorname{Dom}(f)|=\lambda\}$ when $\lambda \leq|\mathscr{U}| / 2$. 4) $K_{\lambda, \mu}^{\mathrm{eq}}=\{E: E$ is an equivalence relation on some $A \subseteq \mathscr{U}$, with $\lambda$ equivalence classes, each of power $\mu\}$.

5) In 4) we can replace " $\mu$ " by " $<\mu$ " if each equivalence class has $<\mu$ elements. Similarly replacing $\lambda$ by " $<\lambda$ ". Similarly $\leq \lambda$, $\leq \mu$.

6) $K_{<\lambda}^{\mathrm{mon}}=\bigcup_{\mu<\lambda} K_{\mu}^{\mathrm{mon}}$ and $K_{<\lambda}^{1-1}=\bigcup_{\mu<\lambda} K_{\mu}^{1-1}$. Similarly with $\leq \lambda$; here the "less than half" is not so important.

7) $K^{\text {mon }}=K_{*}^{\text {mon }}=\{A: A \subseteq \mathscr{U}\}$ and $K^{1-1}=K_{*}^{1-1}=\{f: f$ is a partial one-toone function $\}$.

8) $K_{\lambda, *}^{\mathrm{eq}}=\{E: E$ is an equivalence relation on some $A \subseteq \mathscr{U}$ with $\lambda$-equivalence classes $\}$ and $K_{*,<\mu}^{\mathrm{eq}}=\{E: E$ is an equivalence relation on some $A \subseteq \mathscr{U}$ each equivalence class $<\mu\}$ and $K^{\mathrm{eq}}=K_{\lambda, *}^{\mathrm{eq}}=\{E: E$ is an equivalence relation on some $A \subseteq \mathscr{U}\}$ and lastly $K_{\leq \lambda}^{\mathrm{eq}}=\{E: E$ an equivalence relation on $A \subseteq \mathscr{U},|A| \leq \lambda\}$.

2.2 Remark. More formally $\lambda, \mu$, etc., are functions from $\mathfrak{U}$ to $\mathbb{N}$ which satisfy conditions such as $\lambda(\mathscr{U})<\frac{|\mathscr{U}|}{2}$. We write $\frac{|\mathscr{U}|}{2}$ as shorthand for $\left[\frac{\mathscr{U}}{2}\right]$.

Claims 2.3 through 2.8 are established by similar arguments. To illustrate the technique we prove 2.3(4). If $\varphi\left(x, S_{0}, S_{1}\right)$ denotes " $x \in S_{0} \vee x \in S_{1}$ " then as $S_{1}, S_{2}$ range over subsets of $\mathscr{U}$ with $|\mathscr{U}|<\lambda<\frac{|\mathscr{U}|}{2}$ clearly all sets of cardinality $\kappa, \frac{|\mathscr{U}|}{4} \leq \kappa \leq \frac{|\mathscr{U}|}{2}$ are represented; all sets of cardinality $<\frac{|\mathscr{U}|}{4}$ are represented by $x \in S_{0} \quad \& \quad x \in S_{1}$. (Note this depends on $\left|S_{i}\right| \leq \frac{|\mathscr{U}|}{2}$ ). Finally sets with cardinality between $\frac{|\mathscr{U}|}{2}$ and $|\mathscr{U}|$ are represented by taking compliments.

The choice $\frac{|\mathscr{U}|}{2}$ and $\frac{|\mathscr{U}|}{4}$ is arbitrary. But if $\frac{|\mathscr{U}|}{k}$ for larger $k$ were choice, the union of two sets would have to be replaced by a union of more sets. A lower bound of the form $\frac{|\mathscr{U}|}{k}$ permits the uniform choice of the formula $\varphi$.

2.3 Claim. Let $\lambda \leq \chi<\frac{|\mathscr{U}|}{2}$. Then, uniformly (the choice of the interpreting formula $\varphi$ does not depend on $\mathscr{U}$ ) we have:

0) $Q_{\chi}^{\text {mon }}$ is $\exists_{R}$ for some $R$.

1) $Q_{\lambda}^{\text {mon }} \equiv_{\text {int }} Q_{<\lambda}^{\text {mon }}$ and $Q_{<\lambda}^{\text {mon }} \leq_{\text {int }} Q_{<\chi}^{\text {mon }}$.

2) $Q_{<\mu}^{\mathrm{mon}} \equiv_{\text {int }} Q_{1,<\mu}^{\mathrm{eq}}$.

3) $Q_{\lambda}^{\text {mon }} \equiv_{\text {int }} Q_{\lambda+\lambda}^{\text {mon }}$ if $\lambda(\mathscr{U}) \leq \frac{|\mathscr{U}|}{4}$.

4) If $|\mathscr{U}| / 2 \geq \lambda \geq|\mathscr{U}| / 4$, then $Q_{\lambda}^{\text {mon }} \equiv_{\text {int }} Q^{\text {mon }}$.

5) More generally, for any constants $a$ and $b$, if $\frac{|\mathscr{U}|}{a} \geq \lambda \geq \frac{|\mathscr{U}|}{b}$, then $Q_{\lambda}^{\text {mon }} \equiv_{\text {int }}$ $Q^{\text {mon }}$.

Proof. Straightforward. For 0) recall Notation 1.8(3). For 2) recall Definition 2.1(4). 
2.4 Claim. Let $\lambda \leq \chi$ be as in 2.3.

0) $Q_{\lambda}^{1-1}$ is $\exists_{R}$ for some $R$.

1) $\lambda \leq|\mathscr{U}| / 2 \Rightarrow Q_{\lambda}^{1-1} \equiv_{\text {int }} Q_{\leq \lambda}^{1-1} ; \underline{\text { and }} \chi \leq|\mathscr{U}| / 2 \Rightarrow Q_{<\lambda}^{1-1} \leq_{\text {int }} Q_{<\chi}^{1-1}$.

2) $Q_{<\lambda}^{\operatorname{mon}} \leq$ int $Q_{<\lambda}^{1-1}$.

3) If $\lambda \geq|\mathscr{U}| / 4$, then $Q_{\lambda}^{1-1} \equiv_{\text {int }} Q_{*}^{1-1}$.

4) $Q_{\lambda+\lambda}^{1-1} \equiv_{\text {int }} Q_{\lambda}^{1-1}$ if $\lambda \leq|\mathscr{U}| / 4$.

5) If $R$ is a graph of a partial one-to-one function on $\mathscr{U}, \lambda=\operatorname{Min}\{\mid \operatorname{Dom}(R), R\}$ then $Q_{R} \equiv_{\text {int }} Q_{\lambda}^{1-1}$.

Proof. Straightforward.

2.5 Claim. Let $\lambda \leq \chi$ and $\mu \leq \kappa$ (as in 2.3).

0) $Q_{\lambda, \mu}^{\mathrm{eq}}$ is $\exists_{R}$ for some $R$.

1) If $\chi \leq|\mathscr{U}| / 2, \kappa \leq|\mathscr{U}| / 2$, then $Q_{\lambda, \mu}^{\mathrm{eq}} \leq_{\text {int }} Q_{\chi, \kappa}^{\mathrm{eq}} \equiv_{\mathrm{int}} Q_{\leq \chi, \leq \kappa}^{\mathrm{eq}}$.

1A) $Q_{<\lambda,<\mu}^{\mathrm{eq}} \leq_{\text {int }} Q_{<\chi,<\kappa}^{\mathrm{eq}}$.

2) If $|\mathscr{U}| / 4 \leq \lambda \leq|\mathscr{U}| / 2$ then $Q_{<\lambda}^{\mathrm{eq}} \equiv_{\text {int }} Q_{*, *}^{\mathrm{eq}}$.

3) For equivalence relations $E_{1}, E_{2}$ on $\mathscr{U}$, natural sufficient condition for interpretability works. Similarly for families of equivalence relations.

4) $\exists_{K} \leq_{\text {int }} Q_{\leq \lambda}^{\mathrm{eq}}$ if $(\forall R \in K)\left[|\operatorname{Dom}(R)|^{n(R)} \leq(\lambda-1)^{2}\right]$ and $\lambda \leq|\mathscr{U}| / 2$.

Proof. Left to the reader.

2.6 Definition. 1) $Q_{\lambda}^{\text {ord }}=\{R: R$ a linear order of a subset $A$ of $\mathscr{U}$ of cardinality $\left.^{1} \lambda\right\}$.

2) $Q_{<\lambda}^{\mathrm{ord}}=\bigcup_{\mu<\lambda} Q_{\mu}^{\mathrm{ord}}$.

2.7 Claim. 0) $Q_{\lambda}^{\text {ord }}$ has the form $Q_{R}$.

1) $Q_{\leq \lambda}^{\text {mon }} \leq_{\text {int }} Q_{\lambda}^{\text {ord }}$.

2) If $\mu \times \kappa \leq \lambda$ then $Q_{\mu, \kappa}^{\mathrm{eq}} \leq_{\mathrm{int}} Q_{\lambda}^{\text {ord }}$ and $Q_{<\lambda}^{\mathrm{eq}} \leq_{\text {int }} Q_{\lambda}^{\text {ord }}$.

3) $\mu<\kappa \leq \lambda \Rightarrow Q_{\mu, \kappa}^{\mathrm{eq}} \leq_{1 \text {-int }} Q_{\lambda}^{\text {ord }} \bmod Q_{\leq \lambda}^{\text {ord }}$ and $Q_{\leq \lambda}^{\text {eq }} \leq_{1 \text {-int }} Q_{\lambda}^{\text {ord }} \bmod Q_{\leq \lambda}^{\text {ord }}$.

4) $Q_{\lambda}^{\text {ord }} \leq_{1 \text {-int }} Q_{\lambda}^{1-1} \bmod \mathscr{L}\left(Q_{\leq \mu}^{1-1}\right)$ if $\lambda \leq \mu$.

5) $Q_{\lambda}^{\text {ord }} \leq_{\text {int }} Q_{\lambda, \lambda}^{\mathrm{eq}}$, in fact, one $E_{0} \in Q_{\lambda, \lambda}^{\mathrm{eq}}$, one $E_{1} \in Q_{\left(\lambda^{2}\right), 2}^{\mathrm{eq}}$ and one $P \in Q_{\left(\lambda^{2}\right)}^{\mathrm{mon}}$ suffice.

Proof. Straight.

2.8 Claim. 1) $Q_{K_{1}} \leq_{\text {int }} Q_{K_{2}} \bmod Q_{*}^{1-1}$ is equivalent to $Q_{K_{1}} \leq_{1 \text {-int }} Q_{K_{2}} \bmod$ $Q_{*}^{1-1}$.

2) Similarly for $\leq_{\text {inex }}, \leq_{\exp }$.

\footnotetext{
${ }^{1}$ for the infinite case we $\operatorname{demand} \operatorname{otp}(A, R)=\lambda$
} 
2.9 Definition. For any equivalence relation $E$ on a set $\operatorname{Dom}(E) \subseteq \mathscr{U}$ we define 1) $\mathrm{nu}_{\geq k}(E)$ is the number of equivalence classes of $E$ with $\geq k$ members.

2) $\operatorname{uq}_{k}(E)=\operatorname{Max}\left\{|B|: B \subseteq \mathscr{U}\right.$ and there are $E_{0}, \ldots, E_{k-1} \in Q_{E}[\mathscr{U}]$ such that: $\left.b \neq c \in B \Rightarrow(\exists \ell<k)\left[\left(b E_{\ell} b \equiv \neg c E_{\ell} c\right) \vee\left(b E_{\ell} b \quad \& \quad c E_{\ell} c \& \neg b E_{\ell} c\right)\right]\right\}$.

3) For $x \in \mathscr{U} \backslash \operatorname{Dom}(E)$ let $x / E$ be $\mathscr{U} \backslash \operatorname{Dom}(E)$.

2.10 Claim. 1) $Q_{\mathrm{nu} \geq 2(E)}^{1-1} \leq_{\text {int }} Q_{E}$.

2) $Q_{\mathrm{uq}_{k}(E)}^{1-1} \leq_{\text {int }} Q_{E}$.

Proof. Let $\mathscr{U} \in \mathfrak{U}$.

1) We can find a sequence $\left\langle a_{i}: i<2 \mathrm{nu}_{\geq 2}(E)\right\rangle$ with no repetitions, $a_{i} \in \mathscr{U}$ such that for $i<j$ we have $a_{i} E a_{j} \Leftrightarrow j=i+1 \&$ " $i$ is even". Let $P_{0}=\left\{a_{2 i}: i\right\}, P_{1}=$ $\left\{a_{2 i+1}: i\right\}$. So $P_{0}(x) \& P_{1}(y)$ \& $x E y$ defines a partial one to one function with domain of cardinality $2 \mathrm{nu}_{\geq 2}(E)$. We finish as we can interpret $Q_{\left|P_{0}\right|}^{\operatorname{mon}}$ (or see $3.2(2))$.

2) Easy, too.

2.11 Definition. $Q_{\mu}^{n \text {-ary }}$ is quantifying on $n$-place relation with domain of cardinality $\leq \mu(\mathscr{U})$.

2.12 Claim. 1) For $n$, letting $\bar{x}=\left\langle x_{0}, \ldots, x_{n-1}\right\rangle$ there is a formula $\varphi\left(\bar{x}, F_{0}, \ldots, F_{n-1}\right)$ in monadic logic ( $F_{\ell}$ unary function symbol), such that:

(*) for $\mathscr{U} \in \mathfrak{U}, A \subseteq \mathscr{U}$, an n-place relation $R$ on $A$ we can find a model $M=\left(\mathscr{U}, F_{0}^{M}, \ldots, F_{n-1}^{M}\right)$ and partial one-to-one functions $F_{0}^{M}, \ldots, F_{n-1}^{M}$ from $\mathscr{U}$ to $\mathfrak{U}$ such that $\varphi\left(\bar{x} ; F_{0}^{M}, \ldots, F_{n-1}^{M}\right)$ define $R$ in $M$, where the monadic quantifier is being interpreted as $Q_{<\lambda}^{\mathrm{mon}}, \lambda \geq|R|$ provided that

$\otimes|A|^{n}+|A| \leq|\mathscr{U}|$ or just $|R| \leq|\mathscr{U}|$.

Proof. Let $\left\{\left\langle a_{\ell}^{j}: \ell<n\right\rangle: j<|R|\right\}$ list the $n$-tuples in $R$. Choose $b_{j} \in \mathscr{U} \backslash A$ for $j<|R|$ with no repetition. For each $a \in A$ and $\ell<n$ let $Y_{a}^{\ell}=\left\{j: a_{\ell}^{j}=a\right\}$, so clearly $a^{\prime} \neq a^{\prime \prime} \Rightarrow Y_{a^{\prime}}^{\ell} \cap Y_{a^{\prime \prime}}^{\ell}=\emptyset$ and let $\left\langle j_{a, \ell, k}: k<\left|Y_{a}^{\ell}\right|\right\rangle$ list $Y_{a}^{\ell}$ with no repetition. Define $F_{\ell}^{M}$ by: $F_{\ell}^{M}(a)=b_{j_{a, \ell, 0}}, F_{\ell}^{M}\left(b_{j_{a, \ell, k}}\right)=b_{j_{a, \ell, k+1}}$ except if $Y_{a}^{\ell}=\emptyset$ then $F_{\ell}^{M}\left(a_{j}\right)=a$.

Let

$$
\begin{aligned}
& \varphi\left(\bar{x}, F_{0}, \ldots, F_{n-1}\right)=(\exists z) \bigwedge_{\ell}\left[F_{\ell}\left(x_{\ell}\right)\right. \text { well defined } \\
& \left.\& \neg(\exists y)\left(y \neq x_{\ell} \& F_{\ell}(y)=x_{\ell}\right) \quad \& \theta\left(x_{\ell}, z, F_{\ell}\right)\right]
\end{aligned}
$$

where 


$$
\begin{aligned}
\theta\left(x_{\ell}, z, F_{\ell}\right)=: \forall X & \left(x_{\ell} \in X \quad \&\left(\forall y_{1}, y_{2}\right)\right. \\
& \left.\left(y_{1} \in X \quad \& \quad y_{2}=F_{\ell}\left(y_{1}\right) \& y_{1} \neq z \rightarrow y_{2} \in X\right) \rightarrow z \in X\right) .
\end{aligned}
$$

Those are monadic formulas. Clearly,

$(*)_{1} \varphi$ does not depend on $\mathscr{U}$

$(*)_{2} \quad M \models \theta\left(a, b, F_{\ell}\right)$ iff $b \in\left\{F_{\ell}^{[i]}(a): i\right\}$ where $F_{\ell}^{[0]}(a)=a, F_{\ell}^{[\alpha]}(a)=F_{\ell}\left(F_{\ell}^{[i]}(a)\right)$ (if well defined)

$(*)_{3} \quad M \models " F_{\ell}(a)$ well defined \& $\neg(\exists x)(y \neq F(y)=a)$ iff $a \in A$ [check].

Hence if $\bar{a}=\left\langle a_{\ell}: \ell<n\right\rangle \in A$, by $(*)_{1}+(*)_{2}$ and definition of the $F_{\ell}^{M}$ 's, $\varphi$ and $\theta$ :

$$
\begin{gathered}
M \models \varphi\left[\bar{a}, F_{0}^{M}, \ldots\right] \text { iff for some } z, \bigwedge_{\ell<n}\left(a_{\ell} \in A \& z \in\left\{F_{\ell}^{(i)}(a): i\right\}\right) \\
\text { iff for some } j,\left\langle a_{\ell}: \ell<n\right\rangle=\left\langle a_{\ell}^{j}: \ell<n\right\rangle .
\end{gathered}
$$

2.13 Conclusion. If $\mu_{1}, \mu_{2}$ are functions with domain $\mathfrak{U}, n<\omega$ and $(\forall \mathscr{U} \in \mathfrak{U})\left[\mu_{1}(\mathscr{U})^{n} \leq\right.$ $\left.\mu_{2}(\mathscr{U})\right]$ then

$$
Q_{\mu_{1}}^{n \text {-ary }} \leq_{\text {int }} Q_{\mu_{2}}^{1-1} \bmod Q_{\leq\left(\mu_{1}\right)^{n}}^{\text {mon }}
$$

hence

$$
Q_{\mu_{1}}^{n \text {-ary }} \leq \leq_{\exp } Q_{\mu_{2}}^{1-1}
$$




\section{$\S 2$ Monadic analysis of $\exists_{R}$}

Our aim is to interpret $Q_{\lambda}^{\text {mon }}$ in $\exists_{R}$ for a maximal $\lambda$ and show that except on $\lambda$ elements $R$ is trivial. So continuing later the analysis of $\exists_{R}$, we can instead analyze $\left\{Q_{\lambda}^{\text {mon }}, \exists_{R_{1}}\right\}$ or analyze $\exists_{R_{1}} \bmod Q_{\lambda}^{\text {mon }}$ where $\left|\operatorname{Dom}\left(R_{1}\right)\right| \leq \lambda$ and $\exists_{R_{1}} \leq_{\text {int }} Q_{R}$ and even $\exists_{R_{1}} \leq_{1 \text {-int }} Q_{R} \bmod Q_{\lambda}^{\text {mon }}$. This is made exact below.

3.1 Definition. 1) For any relation $R$ (on $\mathscr{U}$ ) let

$$
\lambda_{0}=\lambda_{0}(R)=\operatorname{Min}\left\{\frac{|\mathscr{U}|}{2}, \lambda_{0}^{\prime}(R)\right\}
$$

where

$$
\begin{aligned}
\lambda_{0}^{\prime}(R)=\operatorname{Min}\{|A|: & A \subseteq \mathscr{U} \text { and for every sequence } \bar{b}, \bar{c} \in \mathscr{U} \\
& \left.(\text { of length } n(R)) \text { we have } \bar{b} \approx_{A} \bar{c} \text { implies } R[\bar{b}] \equiv R[\bar{c}]\right\}
\end{aligned}
$$

where on $\approx_{A}$ see below

2) $\bar{b} \approx_{A} \bar{c}$ means $\bar{b}=\left\langle b_{i}: i\langle n\rangle, \bar{c}=\left\langle c_{i}: i<n\right\rangle\right.$ and

(a) $b_{i} \in A$ iff $c_{i} \in A$

(b) $b_{i} \in A$ implies $b_{i}=c_{i}$

(c) $b_{i}=b_{j}$ iff $c_{i}=c_{j}$.

3) For a set $\Delta$ of formulas $\varphi(\bar{x})$ (where $\varphi$ is a formula, $\bar{x}$ a finite sequence of variables including all variables occuring freely in $\varphi$ ) let

$$
\operatorname{tp}_{\Delta}(\bar{b}, A, \bar{M})=\{\varphi(\bar{x}, \bar{a}): \varphi(\bar{x}, \bar{y}) \in \Delta, \bar{a} \subseteq A \text { and } M=\varphi[\bar{b}, \bar{a}]\} .
$$

We omit $M$ when its identity is clear, and when $M=(\mathscr{U}, R)$ we may write $R$ instead of $M$. We may write $\mathscr{U} \models \varphi[\bar{b}, \bar{a} ; R]$. Replacing $\Delta$ by bs means $\Delta=\{\varphi(\bar{x}): \varphi$ atomic or negation of atomic formula $\}$, here bs stands for basic. We may write $\varphi$ instead $\{\varphi\}$ and $\Delta$ will be always finite.

4) $S_{\Delta}^{m}(A, M)=\left\{\operatorname{tp}_{\Delta}(\bar{b}, A, M): \bar{b} \subseteq M\right.$ and $\left.\ell g(\bar{b})=m\right\}$.

3.2 Remark. 1) Note that $\lambda_{0}(R) \leq \lambda_{0}^{\prime}(R) \leq|\operatorname{Dom}(R)|$.

2) Note that if an equivalence relation $E$ on a subset of $\mathscr{U}$ contains an equivalence class of cardinality $k \geq \frac{|\mathscr{U}|}{2}$ or exactly $k \geq \frac{|\mathscr{U}|}{2}$ singleton classes or $k=|\operatorname{Dom}(E)| \geq$ $\frac{|\mathscr{U}|}{2}$, then $\lambda_{0}^{\prime}(R)=\lambda_{0}(R)=|\mathscr{U}|-k<\frac{|\mathscr{U}|}{2}$. Otherwise, $\lambda_{0}^{\prime}(R)>\frac{|\mathscr{U}|}{2}$ and $\lambda_{0}(R)=$ $\frac{|\mathscr{Q}|}{2}$.

The main result of this section is:

3.3 Theorem. 1) $Q_{\lambda_{0}(R)}^{\text {mon }} \leq_{\text {int }} \exists_{R}$; we mean, of course, uniformly.

2) There is a relation $R_{1}$ on $\mathscr{U}$ with $n\left(R_{1}\right)=n(R)$ and, $\left|\operatorname{Dom}\left(R_{1}\right)\right| \leq \lambda_{0}^{\prime}(R)+n$ such that $\exists_{R} \equiv_{\text {int }}\left\{\exists_{R_{1}}, Q_{\lambda_{0}^{\prime}(R)}^{\text {mon }}\right\}$. In fact, $\exists_{R} \equiv_{1-\text { int }} \exists R_{1} \bmod Q_{\lambda_{0}(R)}^{\text {mon }}$.

The proof is broken into some claims. 
3.4 Claim. Let $R$ be an $n$-place relation on $\mathscr{U}$ such that $n>1$. We can find a set $A$, sequences $\bar{a}_{i}$ and elements $b_{i}, c_{i}$ for $i<i^{*}$, where $i^{*} \geq \frac{\lambda_{0}^{\prime}(R)}{n(R)(n(R)-1)}$ or $i^{*} \geq \frac{|\mathscr{U}|-n(R)}{n(R)(n(R)-1)}$ such that:

(a) $\bar{a}_{i}$ is with no repetition and is $\subseteq A$

(b) $b_{i}, c_{i} \notin A$,

(c) $\left\langle\left(b_{i}, c_{i}\right): i<i^{*}\right\rangle$ is with no repetition, i.e. $i \neq j \Rightarrow b_{i} \neq b_{j} \& c_{i} \neq c_{j}$ \& $b_{i} \neq c_{j}$

(d) $t p_{\mathrm{bs}}\left(\bar{a}_{i}{ }^{\wedge}\left\langle b_{i}\right\rangle, \emptyset, R\right) \neq t p_{\mathrm{bs}}\left(\bar{a}_{i}{ }^{\wedge}\left\langle c_{i}\right\rangle, \emptyset, R\right)$, that is for some atomic formula $\varphi$ we have $(\mathscr{U}, R) \models \varphi\left(\bar{a}_{i}, b_{i}\right) \equiv \neg \varphi\left(\bar{a}_{i}, c_{i}\right)$.

Proof. We try to choose by induction on $i,\left\langle A_{\ell}^{i}: \ell<n(R)\right\rangle,\left\langle\bar{a}_{i}, b_{i}, c_{i}\right\rangle$ and $\ell(i)<$ $n(R)$ such that:

(i) $\ell<k<n(R) \Rightarrow A_{\ell}^{i} \cap A_{k}^{i}=\emptyset$

(ii) $A_{\ell}^{i} \subseteq A_{\ell+1}^{i}$ and $A_{\ell}^{0}=\emptyset$

(iii) $\bar{a}_{i}{ }^{\wedge}\left\langle b_{i}\right\rangle^{\wedge}\left\langle c_{i}\right\rangle$ is with no repetition and has length $\leq n(R)+1$

(iv) $\operatorname{tp}_{\mathrm{bs}}\left(\bar{a}_{i}{ }^{\wedge}\left\langle b_{i}\right\rangle, \emptyset, R\right) \neq \operatorname{tp}_{\mathrm{bs}}\left(\bar{a}_{i}{ }^{\wedge}\left\langle c_{i}\right\rangle, \emptyset, R\right)$, that is for some atomic formula $\varphi(\bar{x}, y)$ (so gotten from $R\left(x_{0}, \ldots, x_{n(R)-1}\right)$ by substitution) we have $\varphi\left(\bar{a}_{i}, b_{i}\right) \equiv \neg \varphi\left(\bar{a}_{i}, c_{i}\right)$

(v) $b_{i}, c_{i} \notin \cup\left\{A_{\ell}^{i}: \ell<n(R)\right\}$

(vi) $\ell(i)=\operatorname{Min}\left\{\ell: \bar{a}_{i} \cap A_{\ell}^{i}=\emptyset\right\}$

(vii) $A_{\ell(i)}^{i+1}=A_{\ell(i)}^{i} \cup\left\{b_{i}, c_{i}\right\}$

(viii) $A_{\ell}^{i+1}$ is $A_{\ell}^{i} \cup\left\{\operatorname{Rang}\left(\bar{a}_{i}\right) \backslash \bigcup_{m} A_{m}^{i}\right\}$

if $(\ell=0 \quad \& \quad \ell(i)>0) \vee(\ell=1 \quad \& \quad \ell(i)=0)$

(ix) $A_{\ell}^{i+1}=A_{\ell}^{i}$ in the other cases.

So for some $i=i(*)$ we cannot continue; we claim that $A=: \bigcup_{\ell} A_{\ell}^{i}$ has cardinality $\geq \lambda_{0}^{\prime}(R)$ or $\geq|\mathscr{U}|-n(R)$.

Why? Otherwise by the definition of $\lambda_{0}^{\prime}(R)$ there are sequence $\bar{b}, \bar{c}$ from $\mathscr{U}$ of length $n(R)$ such that $\bar{b} \approx_{A} \bar{c}$ but $\bar{b} \in R \equiv \bar{c} \notin R$. Hence we can find sequences $\bar{b}^{\prime}, \bar{c}^{\prime}$ from $\mathscr{U}$ of the same length $\leq n(R)$, each with no repetitions such that $\bar{b}^{\prime} \approx_{A} \bar{c}^{\prime}$ but for some $\varphi=R(\bar{x})=R\left(x_{i_{0}}, x_{i_{1}}, \ldots, x_{i_{n_{R}}-1}\right), \ell g(\bar{x})=\ell g\left(\bar{b}^{\prime}\right)=\ell g\left(\bar{c}^{\prime}\right)$ we have $\varphi\left(\bar{b}^{\prime}\right) \& \neg \varphi\left(\bar{c}^{\prime}\right)$. Now we can find $k$ and $\bar{d}_{0}, \ldots, \bar{d}_{k}$ such that: $\bar{d}_{0}=\bar{b}^{\prime}, \bar{d}_{k}=\bar{c}^{\prime}$, and $\bar{d}_{\ell}$ is with no repetitions, $\ell<k \Rightarrow \bar{d}_{\ell} \approx_{A} \bar{d}_{\ell+1}$ and $\ell<k \Rightarrow(\exists ! i) d_{\ell, i} \neq d_{\ell+1, i}$; here we use the assumption toward contradiction $|A| \leq|\mathscr{U}|-n$.

So for some $\ell<k$ we have $\varphi\left(\bar{d}_{\ell}\right) \& \neg \varphi\left(\bar{d}_{\ell+1}\right)$. Now let $r$ be such that $d_{\ell, r} \neq d_{\ell+1, r}$, so without loss of generality $r=\ell g(\bar{x})-1$, let $\bar{a}_{i(*)}=\bar{d}_{\ell, r} \uparrow\left(\ell g\left(\bar{b}_{\ell}\right)-1\right), b_{i(*)}=$ $d_{\ell, r}, c_{i(*)}=d_{\ell+1, r}$. Clearly they are as required in clause (iii) $+(\mathrm{iv})$, now $\ell(i(*))$ is well defined by clause (vi) as $\left|\operatorname{Rang}\left(\bar{a}_{i}\right)\right| \leq n(R)-1$, so $\left|\operatorname{Rang}\left(\bar{a}_{i}\right) \cap\left(\bigcup_{\ell} A_{\ell}^{i}\right)\right|<n(R)$.

Now we can define $A_{\ell}^{i(*)+1}$ for $\ell<n(R)$ by clauses (vii), (viii) and (ix). Trivially, 
clauses (i) and (ii) hold, and we get a contradiction to the choice of $i(*)$. So really $|A| \geq \lambda_{0}^{\prime}(R)$ or $|A| \geq|\mathscr{U}|-n(R)$.

Now note that $\left|\bigcup_{\ell} A_{\ell}^{i(*)}\right| \leq(n(R)+1) \times i(*)$, by clauses (vii), (viii), (ix) so $i(*) \geq\left|\bigcup_{\ell} A_{\ell}^{i(*)}\right| /(n(R)+1)$ and for some $\ell$ we have $|\{i<i(*): \ell(i)=\ell\}| \geq$ $i(*) / n(R)$. So if $\lambda_{0}^{\prime}(R)<|\mathscr{U}|-n(R)$ we get $|\{i<i(*): \ell(i)=\ell\}| \geq i(*) / n(R) \geq$ $\lambda_{0}^{\prime}(R) /\left((n(R)+1)(n(R))\right.$. If $\lambda_{0}^{\prime}(R) \geq|\mathscr{U}|-n(R)$ we get $|\{i<i(*): \ell(i)=\ell\}| \geq$ $(|\mathscr{U}|-n(R)) /(n(R)(n(R)+1))$. So renaming we are done.

3.5 Claim. There is a formula $\varphi^{*}=\varphi^{*}(x, \bar{y} ; R)$, in first order logic, of course, such that:

(*) if ( $R$ is an $n(R)$-place relation on $\mathscr{U}$ and) $\lambda_{0}^{\prime}(R)<\frac{2}{3}|\mathscr{U}|$, then $\varphi^{*}$ exemplifies $Q_{\lambda_{0}(R)} \leq \leq_{\text {int }} Q_{R}$ even $Q_{\lambda_{0}(R)} \leq \leq_{1 \text {-int }} Q_{R}$ specifically, for some

$\bar{d}$ we have $\left\{a:(\mathscr{U}, R)=\varphi^{*}(a, \bar{d}, R)\right\}$ has $\lambda_{0}(R)$ members.

Proof. Without loss of generality $\frac{1}{3}|\mathscr{U}|>n(R)^{2}+n(R)$.

Let $A \subseteq \mathscr{U}$ be a set of power $\lambda_{0}^{\prime}(R)$ such that $\bar{b} \approx_{A} \bar{c}$ implies $R[\bar{b}] \equiv R[\bar{c}]$. As $|\mathscr{U}|-\lambda_{0}^{\prime}(R)$ is large enough, we can find pairwise distinct $d_{i} \in \mathscr{U} \backslash A$ for $i<n(R)^{2}$. Define $\bar{d}=\left\langle d_{i}: i<n(R)^{2}\right\rangle$ and $\varphi^{*}(x, \bar{d}, R)=\bigvee\left\{\left(\exists y_{0}, \ldots, y_{k-1}\right)\right.$ [the elements $y_{0}, \ldots, y_{k-1}, x$ are pairwise distinct and for any $m$ if the elements $y_{0}, \ldots, y_{k-1}, d_{m}, x$ are pairwise distinct and $\left.\varphi\left(x, y_{0}, \ldots, y_{k-1}\right) \equiv \neg \varphi\left(d_{m}, y_{0}, \ldots, y_{k-1}\right)\right]: \varphi=\varphi\left(z_{0}, \ldots, z_{k}, R\right)$ is an atomic formula in $L(R)$ (so $k+1 \leq n(R))$ and $m<n(R)^{2}$, so $m, k$ are natural numbers $\}$. By the choice of $A$ we have $x \notin A \Rightarrow \neg \varphi^{*}(x, \bar{d}, R)$, hence $B=:\left\{x \in \mathscr{U}: \mathscr{U} \models \varphi^{*}[x, \bar{d}, R]\right\}$ is a subset of $A$. Clearly $Q_{|B|}^{\text {mon }} \leq_{\text {int }} \exists_{R}$ (uniformly); hence it suffices to prove $|B|=\lambda_{0}^{\prime}(R)$ which follows if we show

$(*)$ if $\bar{b} \cong_{B} \bar{c}$ then $R[\bar{b}] \equiv R[\bar{c}]$.

For this it suffices to prove

$(* *)$ if $\varphi(\bar{x}, R) \in L(R)$ is atomic, $\bar{b}, \bar{c}$ are sequences of length $\ell g(\bar{x}) \leq n(R)$ without repetition then $\bar{b} \cong_{B} \bar{c}$ implies $\varphi(\bar{b}, R) \equiv \varphi(\bar{c}, R)$.

To prove $(* *)$, by reordering the sequences we let $\bar{b}^{\wedge} \bar{c}_{0}, \bar{b}^{\wedge} \bar{c}_{1}$ be sequences from $\mathscr{U}$, without repetition, $\bar{b} \subseteq B, \bar{c}_{0}, \bar{c}_{1}$ disjoint to $B$; by the transitivity of $\equiv$, without loss of generality $\bar{c}_{1}$ is disjoint to $\bar{d}$. Now for some $i,\left\langle d_{i}, d_{i+1}, \ldots, d_{i+k-1}\right\rangle$ (where $\left.k=\ell\left(\bar{c}_{0}\right)\right)$ is disjoint to $\bar{c}_{0}$ (and obviously to $\bar{c}_{1}$ ).

Now we shall prove that for every atomic $\varphi(\bar{x}, \bar{y}, R), \ell g(\bar{x})=k, \ell g(\bar{y})=\ell g(\bar{b})$ we have $\models \varphi\left(\bar{c}_{\ell}, \bar{b}, R\right) \equiv \varphi\left(\left\langle d_{i}, \ldots, d_{i+k-1}\right\rangle, \bar{b}, R\right)$ thus finishing. For this we define $\bar{c}_{\ell, m}(m \leq k)$ such that each $\bar{c}_{\ell, m}$ is with no repetitions, disjoint to $B \cup \bar{b}$ and $\bar{c}_{\ell, 0}=\bar{c}_{\ell}, \bar{c}_{\ell, k}=\left\langle d_{i}, \ldots, d_{i+k-1}\right\rangle, \bar{c}_{\ell, m+1}, \bar{c}_{\ell, m}$ are distinct in one place only. By the definition of $B$ (and $\varphi$ ) for every atomic $\varphi(\bar{x}, \bar{y}, R)$ we have $\models \varphi\left(\bar{c}_{\ell, m}, \bar{b}, R\right) \equiv$ $\varphi\left(\bar{c}_{\ell, m+1}, \bar{b}, \bar{R}\right)$ so we finish easily. (Being more careful, e.g. $\frac{1}{9}|\mathscr{U}| \geq n(R)$ suffices).

Remark. Note that definition of $\lambda_{0}$ applies to any relation, in particular, the relation being defined by a formula so we may freely speak at $\lambda_{0}(\psi)$ or $\lambda_{0}(\psi(\bar{x}))$. 
3.6 Claim. $Q_{\leq \lambda_{0}(R)}^{\operatorname{mon}} \leq_{\text {int }} Q_{R}$.

Proof. If we can replace in $R$ some variables by constants or other variables having at least one equality getting a relation $R^{\prime}$ such that $\lambda_{0}\left(R^{\prime}\right) \geq \operatorname{Min}\left\{\lambda_{0}(R), \frac{1}{7 n(R)}|\mathscr{U}|\right\}$ we do it: or in other words we are inducting on $n(R) \geq 1$.

Case 1: $n(R)=1$.

So $R$ is unary; now note that each of the sets $A=R, A^{\prime}=\mathscr{U} \backslash R$ can serve in the definition of $\lambda_{0}^{\prime}(R)$, hence

$$
\lambda_{0}^{\prime}(R) \leq \operatorname{Min}\left\{|A|,\left|A^{\prime}\right|\right\}=\operatorname{Min}\{R,|\mathscr{U} \backslash R|\} \leq \frac{|\mathscr{U}|}{2}
$$

so we are clearly done.

Case 2: $n(R)>1$.

If $\lambda_{0}^{\prime}(R) \leq \frac{2}{3}|\mathscr{U}|$ we can interpret $Q_{\leq \lambda_{0}(R)}^{\text {mon }}$ by 3.5 , as it suffices to show that at least one of several $\varphi$ 's interpret. So assume $\lambda_{0}^{\prime}(R)>\frac{2}{3}|\mathscr{U}|$. Hence $\lambda_{0}(R)=$ $\frac{|\mathscr{U}|}{2}$ and we shall prove that we can interpret $Q_{<\lambda_{0}(R)}^{\operatorname{mon}}$, for this it is enough if we can show that we can interpret $Q_{\leq\left[\frac{|\mathscr{Q}|}{2^{2^{(R)}}}\right]}^{\mathrm{mon}}$. For this is enough to find first order $\theta\left(\bar{x}_{1}, \bar{y}_{1}, R\right), \ldots, \theta_{k}\left(\bar{x}_{k}, \bar{y}_{k}, R\right)$ with the $k$ and $\theta_{\ell}$ depending only on $n(R)$ and not on $|\mathscr{U}|$ such that $\ell g\left(\bar{x}_{\ell}\right)<n(R)$ and for some $\ell \in\{1, \ldots, k\}$ and $\bar{b} \in{ }^{\ell g\left(\bar{y}_{\ell}\right)} \mathscr{U}$, we have

$$
\frac{|\mathscr{U}|}{2^{2^{n(R)}}} \leq \lambda_{0}^{\prime}\left(\theta_{\ell}(-, \bar{b}, R)\right)
$$

For any $\ell<k<n(R)$ we can consider the formula $R_{\ell, k}\left(x_{0}, \ldots, x_{n(R)-1}\right)=$ $R\left(x_{0}, \ldots, x_{n(R)-1}\right) \quad \& \quad x_{\ell}=x_{k}$ and $R^{*}=R\left(x_{0}, \ldots, x_{n-1}\right) \quad \& \quad \bigwedge_{\ell<k} x_{\ell} \neq x_{k}$. Easily $\lambda_{0}^{\prime}(R) \leq \lambda_{0}^{\prime}\left(R^{*}\right)+\sum_{\ell<k<n(R)} \lambda_{0}^{\prime}\left(R_{\ell, k}\right)$. Now if for some $\ell<k, \lambda_{0}\left(R_{\ell, k}\right) \geq$ $\frac{|\mathscr{U}|}{3 n(R)(n(R-1))}$ we are done by the induction hypothesis. So we can assume $\lambda_{0}^{\prime}\left(R^{*}\right) \geq$ $\frac{|\mathscr{U}|}{3 n(R)(n(R)-1)}$ hence by the above without loss of generality $\lambda_{0}^{\prime}\left(R^{*}\right) \geq \frac{2}{3}|\mathscr{U}|$, so we can assume

$$
(*)_{0} \quad R=R^{*} .
$$

So atomic formulas not equivalent to a fix truth value except equality are just $R\left(\ldots, x_{\sigma(\ell)}, \ldots\right)$ for $\sigma \in \operatorname{Per}(n(R))$.

Let $A, \bar{a}_{i}, b_{i}, c_{i}$ for $i<j^{*}=(|\mathscr{U}|-n(R)) /(n(R)(n(R)-1))$ be as guaranteed by 3.4. For some atomic $\varphi=\varphi(\bar{x}, y)=\varphi(\bar{x}, y, R)$ we have $\left|\left\{i: \varphi\left(\bar{a}_{i}, b_{i}\right) \wedge \neg \varphi\left(\bar{a}_{i}, c_{i}\right)\right\}\right| \geq$ $j^{*} / n(R)$, by $(*)_{0}$. Without loss of generality this occurs for $i<j^{*} / n(R)$. For $i \leq j^{*}$ let $F_{i}$ be the permutation of $\mathscr{U}$, interchanging $b_{j}, c_{j}$ for $j<i$ and being the identity otherwise. Let $R_{i}=F_{i}^{\prime \prime}(R), \psi_{i}=\psi_{i}(\bar{x}, y)=:\left[\psi\left(\bar{x}, y, R, R_{i}\right)=\varphi(\bar{x}, y, R) \&\right.$ $\left.\neg \varphi\left(\bar{x}, y, R_{i}\right)\right]$ so

$$
(*) \psi_{j}\left(\bar{a}_{i}, b_{i}\right) \& \neg \psi_{j}\left(\bar{a}_{i}, c_{i}\right) \text { if } i<j .
$$


So by the definition of $\lambda_{0}^{\prime}(-)$ we have $\lambda_{0}^{\prime}\left(\psi_{j}\right) \geq j$ for $j \leq j^{*} / n(R)$, where we consider $\psi_{j}$ as a $(\ell g(\bar{x})+1)$-place relation.

[Why? If $A \subseteq|\mathscr{U}|,|A|<j$ exemplifies the failure of this assertion (by the definition of $\left.\lambda_{0}^{\prime}(R)\right)$ then $w=:\left\{i<j: A \cap\left\{b_{i}, c_{i}\right\} \neq \emptyset\right\}$ has $\leq|A|$ members, so choose $i \in j \backslash w$, now $\mathscr{U} \models \psi_{j}\left(\bar{a}_{i}, b_{i}, c_{i}\right) \& \neg \psi_{j}\left(\bar{a}_{i}, c_{i}, b_{i}\right)$, (holds by $\left.(*)\right)$ contradict the choice of $A$.] So if $\bigvee_{j}\left[|\mathscr{U}| / 3 \leq \lambda_{0}^{\prime}\left(\psi_{j}\right)<\frac{2}{3}|\mathscr{U}|\right]$ we are done; hence assume not.

If for every $j$ we have $\lambda_{0}^{\prime}\left(\psi_{j}\right)<\frac{|\mathscr{U}|}{3}$, then we get $\left[\frac{|\mathscr{U}|-n(R)}{n(R)^{2}(n(R)-1)}\right]=\left[j^{*} / n(R)\right] \leq$ $\lambda_{0}^{\prime}\left(\psi_{\left[j^{*} / n(R)\right]}\right) \leq \frac{|\mathscr{U}|}{3}$, so we easily finish by 3.5 .

Also $\lambda_{0}^{\prime}\left(\psi_{0}\right)=\lambda_{0}^{\prime}(\emptyset)=0$ as $R_{0}=R$ so $\mathscr{U} \models \varphi(\bar{x}, y, R) \equiv \varphi\left(\bar{x}, y, R_{0}\right)$ hence $\mathscr{U} \models$ $\neg \psi_{0}\left(\bar{x}, y, R, R_{0}\right)$. Without loss of generality $\varphi$ is $R$. So the bad case is that for some $j$ we have $\lambda_{0}^{\prime}\left(\psi_{j}\right)<\frac{1}{3}|\mathscr{U}|$ and $\lambda_{0}^{\prime}\left(\psi_{j+1}\right) \geq \frac{2}{3}|\mathscr{U}|$. Let $B^{*} \subseteq \mathscr{U}$ exemplify $\lambda_{0}^{\prime}\left(\psi_{j}\right)<$ $\frac{1}{3}|\mathscr{U}|$. Let for $\ell<n(R), \theta_{\ell}\left(x_{0}, \ldots, x_{n(R)-2}, y, R\right)=R\left(x_{0}, \ldots, x_{\ell-1}, y, x_{\ell}, \ldots, x_{n(R)-2}\right)$ and on $\theta_{\ell}\left(\left\langle x_{m}: m<n(R)-1\right\rangle, c_{j} ; R_{j}\right)$ we apply our induction hypothesis as its arity is $\ell g(\bar{x})$ which is at most $n(R)-1$ (see the beginning of the proof) hence $\lambda_{0}^{\prime}\left(\theta_{\ell}\left(\left\langle x_{m}: m<n(R)-1\right\rangle, c_{j}, R\right)\right) \leq \frac{1}{7 n(R)}|\mathscr{U}|$ and let $B_{\ell} \subseteq \mathscr{U}$ exemplify it. Similarly let $B_{\ell}^{\prime} \subseteq \mathscr{U}$ exemplify $\lambda_{0}^{\prime}\left(\theta_{\ell}\left(\left\langle x_{m}: m<n(R)-1\right\rangle, b_{j}, R\right)\right) \leq \frac{1}{7 n(R)}$. Let $B=B^{*} \cup \bigcup_{\ell<n(R)} B_{\ell} \cup \bigcup_{\ell<n(R)} B_{\ell}^{\prime} \cup\left\{b_{j}, c_{j}\right\}$. Now $B$ is a subset of $\mathscr{U}$ with $<\left(\frac{2}{7}+\frac{1}{3}\right)|\mathscr{U}|+2<\frac{2}{3}|\mathscr{U}|$ elements. By the definition of $\psi_{j}, \psi_{j+1}$ and $(*)_{0} \operatorname{such} B$ exemplifies $\lambda_{0}^{\prime}\left(\psi_{j+1}\right)<\frac{2}{3}|\mathscr{U}|$, contradiction.

We have implicitly used:

3.7 Claim. If $R$ is a Boolean combination of $R_{0}, \ldots, R_{n-1} \underline{\text { then }} \lambda_{0}^{\prime}(R) \leq \sum_{\ell<n} \lambda_{0}^{\prime}\left(R_{\ell}\right)$ hence $\lambda_{0}(R) \leq \sum_{\ell<n} \lambda_{0}\left(R_{\ell}\right)$.

Proof. If $A_{\ell}$ witnesses the value $\lambda_{0}^{\prime}\left(R_{\ell}\right)$ then $A=\bigcup_{\ell<k} A_{\ell}$ witnesses $\lambda_{0}^{\prime}(R) \leq|A| \leq$ $\sum_{i<k}\left|A_{\ell}\right|$

Now we turn to 3.3

Proof of 3.3(1).

Immediate by $3.5,3.6$.

Proof of 3.3(2). Let $d_{i}$ (for $i<n(R)$ ) be distinct elements of $\mathscr{U} \backslash A$ where $A$ exemplifies $\lambda_{0}^{\prime}(R)$ as if $\lambda_{0}^{\prime}(R)+n \geq|\mathscr{U}|$ then we can choose $R_{1}=R$. Of course, we can concentrate on the case $n(R)>1$. Let $R_{1}=R \uparrow\left(A \cup\left\{d_{i}: i<n(R)\right\}\right.$. So $\left\langle a_{1}, \ldots, a_{n}\right\rangle \in R$ iff for some $\left\langle a_{1}^{\prime}, \ldots, a_{n}^{\prime}\right\rangle \in R_{1}$ we have $\left\langle a_{1}, \ldots, a_{n}\right\rangle \approx_{A}\left\langle a_{1}^{\prime}, \ldots, a_{n}^{\prime}\right\rangle$ 
and $\bigwedge_{\ell}\left[a_{\ell}^{\prime} \notin A \rightarrow \bigvee_{m}\left[a_{\ell}^{\prime}=d_{m}\right]\right.$, so we can define $R_{1}$ from $R$ and $R$ from $R_{1}$ by a quantifier free formula using the unary relation $A$ and individual constants $d_{0}, d_{1}, \ldots, d_{n(R)-1}$. Hence $\exists_{R} \leq_{1 \text {-int }} \exists_{R_{1}} \bmod Q_{\lambda_{0}^{\prime}(R)}^{\text {mon }}$ but $\lambda_{0}^{\prime}(R) \leq 2 \lambda_{0}(R)$ so $\exists_{R} \leq{ }_{1 \text {-int }} \exists_{R_{1}} \bmod Q_{\lambda_{0}(R)}^{\text {mon }}$.

Also easily $\left\{\exists_{R_{1}}, Q_{\lambda_{0}(R)}^{\text {mon }}\right\} \leq_{1-\text { int }} \exists_{R}$.

We can get the parallel result for $Q_{K}$.

3.8 Definition. Let $\lambda_{0}(K)=\operatorname{Min}\left\{\lambda: R \in K \Rightarrow \lambda_{0}(R)<\lambda\right\}$ note that the minimum is taken for each $\mathscr{U} \in \mathfrak{U}$ separately.

3.9 Theorem. 1) $Q_{\leq \lambda_{0}(K)}^{\operatorname{mon}} \leq_{\text {int }} \exists_{K}$.

2) There is $K_{1}, n\left(K_{1}\right)=n(K)$ such that

(a) $\exists_{K} \equiv_{\mathrm{int}}\left\{\exists_{K_{1}}, Q_{<\lambda_{0}(K)}^{\mathrm{mon}}\right\}$

(b) $R \in K \Rightarrow|\operatorname{Dom}(K)|<\lambda_{0}(K)$

(c) $\exists_{K} \equiv_{1 \text {-int }} \exists_{K_{1}} \bmod Q_{<\lambda_{0}(K)}^{\operatorname{mon}}$.

Proof. Immediate by the uniformity of our results.

3.10 Discussion: The interpretation here uses first order formulas of low complexity but use several copies of $R$. We may wonder if we can just use one copy of $R$ by complicating the formula. Now if $R$ is a connected graph every node having a valency $\leq m<<|\mathscr{U}|$, we see that not. But we can prove that the general situation in the problematic case is not far from this (similar to a model of a strongly minimal theory, a local version). Also in general 2 copies of $R$ suffice. 


\section{$\S 3$ The One-TO-ONE FUnCtion ANALYSIS}

The aim of this section is similar to the previous one, going one step further, i.e. we want to analyze $\exists_{R}$, interpreting in it $Q_{\lambda}^{1-1}$ for a maximal $\lambda$, hoping that "the remainder" has domain $\leq \lambda$.

4.1 Definition. Let $\lambda_{1}(R)$ be $\operatorname{Max}\left\{\left|\left\{\operatorname{tp}_{\mathrm{bs}}(a, A, R): a \in \mathscr{U} \backslash A\right\}\right|: A \subseteq \mathscr{U}\right\}$. (On tpbs see $3.1(3))$.

4.2 Fact. $\lambda_{1}(R) \leq \lambda_{0}^{\prime}(R)+1$ and if equality holds then $\lambda_{1}(R) \leq 2^{2^{n(R)^{2}}}$.

Proof. Straight, assume $A_{0}$ exemplifies $\lambda_{0}^{\prime}(R)$ and let $A \subseteq \mathscr{U}$. Then $a, b \in(\mathscr{U} \backslash A) \backslash A_{0} \Rightarrow$ $\operatorname{tp}_{\mathrm{bs}}(a, A, R)=\operatorname{tp}_{\mathrm{bs}}(b, A, R)$ by the choice of $A_{0}$ hence $\mid\left\{\operatorname{tp}_{\mathrm{bs}}(a, A, R): a \in\right.$ $\mathscr{U} \backslash A\}|\leq| A_{0} \backslash A|+1 \leq| A_{0} \mid+1=\lambda_{0}^{\prime}(R)+1$. Next assume that equality holds, so necessarily $\left|A_{0} \backslash A\right|=\left|A_{0}\right|$ hence $A \cap A_{0}=\emptyset$; now choose $A^{\prime} \subseteq A$ with $\operatorname{Min}\{n(R)-1,|A|\}$ elements. By the choice of $A_{0}$, if $b, c \in \mathscr{U} \backslash A$ then

$$
\operatorname{tp}_{\mathrm{bs}}(b, A, R)=\operatorname{tp}_{\mathrm{bs}}(c, A, R) \Leftrightarrow \operatorname{tp}_{\mathrm{bs}}\left(b, A^{\prime}, R\right)=\operatorname{tp}_{\mathrm{bs}}\left(c, A^{\prime}, R\right) .
$$

[Why? $\Rightarrow$ holds as $A^{\prime} \subseteq A$; next we shall prove $\Rightarrow$. This suffices so assume $\operatorname{tp}_{\mathrm{bs}}\left(b, A^{\prime}, R\right)=\operatorname{tp}_{\mathrm{bs}}\left(c, A^{\prime}, R\right)$. So let $\varphi(x, \bar{y}, R)$ be an atomic formula (i.e. a substitution in $R\left(x_{0}, \ldots, x_{n(R)-j}\right)$, so $\left.\ell g(\bar{y})+1 \leq n(R)\right)$ and let $\bar{a}_{1}$ be a sequence of length $\ell g(\bar{y})$ from $A$, we shall show that $\varphi\left(b, \bar{a}_{1}, R\right) \equiv \varphi\left(c, \bar{a}_{1}, R\right)$, this suffices. If $|A|<n(R)$, then $A^{\prime}=A$ and we are done, so assume $|A| \geq n(R)$.

We can find a sequence $\bar{a}_{2}$ from $A^{\prime}$ which realizes the same equality type as $\bar{a}_{1}$ (because $\left.\ell g\left(\bar{a}_{1}\right)=\ell g(\bar{y}) \leq n(R)-1=\left|A^{\prime}\right|\right)$. Now by our assumption $\varphi\left(b, \bar{a}_{2}, R\right) \equiv$ $\varphi\left(c, \bar{a}_{2}, R\right)$ (that is as $\left.\operatorname{tp}_{\mathrm{bs}}\left(b, A^{\prime}, R\right)=\operatorname{tp}_{\mathrm{bs}}\left(c, A^{\prime}, R\right)\right)$, so to get our desired $\varphi\left(b, \bar{a}_{1}, R\right) \equiv$ $\varphi\left(c, \bar{a}_{1}, R\right)$ it suffices to prove $\varphi\left(b, \bar{a}_{1}, R\right) \equiv \varphi\left(b, \bar{a}_{2}, R\right)$ and $\varphi\left(c, \bar{a}_{1}, R\right) \equiv \varphi\left(c, \bar{a}_{2}, R\right)$. But on both $b$ and $c$ we just assume they are in $\mathscr{U} \backslash A$, so by symmetry it is enough to show $\varphi\left(b, \bar{a}_{1}, R\right) \equiv \varphi\left(b, \bar{a}_{2}, R\right)$. Now as $\bar{a}_{1}, \bar{a}_{2}$ are included in $A$ and have the same equality type (over the $\emptyset$ ), by the choice of $A_{0}$ and as $A_{0} \cap A=\emptyset$ necessarily $\bar{a}_{1}, \bar{a}_{2}$ realizes the same equality type over $\mathscr{U} \backslash A$, so as $b \in \mathscr{U} \backslash A$ we have $\left.\varphi\left(b, \bar{a}_{1}, R\right) \equiv \varphi\left(b, \bar{a}_{2}, R\right).\right]$

Hence $\lambda_{1}(R) \leq\left|\left\{\operatorname{tp}_{\mathrm{bs}}\left(b, A^{\prime}, R\right): b \in \mathscr{U}\right\}\right| \leq 2^{|\Phi|}$ where $\Phi$ is the set of atomic formulas $\varphi(x, \bar{a})$ such that $\bar{a} \subseteq A^{\prime},|\Phi| \leq n(R) \times(n(R)-1)^{n(R)-1} \leq 2^{n(R)^{2}}$.

4.3 Claim. $Q_{\lambda_{1}(R)}^{1-1} \leq_{\text {int }} \exists_{R}$; of course uniformly.

Proof. Suppose $h$ is a one-to-one, one place partial function from $\mathscr{U}$ to $\mathscr{U}$ with $\lambda=|\operatorname{Dom}(h)| \leq \lambda_{1}(R)$ and $\lambda \leq \frac{1}{n(R)+1}|\mathscr{U}|$ (we use freely 2.4). Let $A \subseteq \mathscr{U}$ be such that $\left\{\operatorname{tp}_{\mathrm{bs}}(a, A, R): a \in \mathscr{U} \backslash A\right\}$ has cardinality $\lambda_{1}(R)$. So we can find $a_{i} \in \mathscr{U} \backslash A$ (for $i<\lambda$ ) such that $\operatorname{tp}_{\mathrm{bs}}\left(a_{i}, A, R\right)$ are pairwise distinct. Retaining the last sentence (by not necessarily the original demand on $A$ ) without loss of generality $|A| \leq|\mathscr{U}|-\lambda-\lambda$.

[Why? Just for each $i=1, \ldots, \lambda-1$ choose $\bar{d}_{i} \subseteq A$ of length $<n(R)$ such that 
$\left\langle\operatorname{tp}_{\mathrm{bs}}\left(a_{j}, \bigcup_{\ell=1}^{i} \bar{d}_{\ell}\right): j \leq i\right\rangle$ is with no repetitions so without loss of generality $|A| \leq$ $(n(R)-1) \times \lambda$ and compute.] Let $h=\left\{\left\langle b_{i}, c_{i}\right\rangle: i<\lambda\right\}$, without loss of generality $b_{i}, c_{i} \notin A$ (just permute $R$, i.e. using an isomorphic $R^{\prime}$ ) and we can find $F_{1}, F_{2}$ permutation of $\mathscr{U}$ which are the identity on $A$ such that $F_{1}\left(a_{i}\right)=$ $b_{i}, F_{2}\left(a_{i}\right)=c_{i}$. Let $R_{1}=F_{1}(R)$ and $R_{2}=F_{2}(R)$ and define the monadic relations $P_{0}=A, P_{1}=\left\{b_{i}: i<\lambda\right\}, P_{2}=\left\{c_{i}: i<\lambda\right\}$ (all of cardinality $\leq \lambda_{0}(R)$ ). Let $\varphi\left(x, y, P_{0}, P_{1}, P_{2}, R_{1}, R_{2}\right)$ "say" that for every atomic $\psi(x, \bar{z}, R) \in L(R)$ and $\bar{t} \in P_{0}$ we have: $\varphi\left(x, \bar{t}, R_{1}\right) \equiv \varphi\left(y, \bar{t}, R_{2}\right)$ and $P_{1}(x), P_{2}(y)$. Clearly $\varphi$ defines $h . \quad \square_{4.3}$

4.4 Lemma. Assume $\lambda_{1}(R) \times n(R)^{2}+n(R)<|\mathscr{U}|$. For any set $A \subseteq \mathscr{U}$, let $E_{A}$ be the following equivalence relation on $\mathscr{U}: t p_{\mathrm{bs}}(a, A, R)=t p_{\mathrm{bs}}(b, A, R)$. For any $A \subseteq \mathscr{U}$ and $\bar{C}=\left\langle C_{\ell}: \ell<k\right\rangle$ such that $C_{\ell} \subseteq \mathscr{U}$ let $E_{A, \bar{C}}$ be the following equivalent relation on $\mathscr{U}: a E_{A, \bar{C}} b$ iff $a E_{A} b \& \bigwedge_{\ell} a \in C_{\ell} \equiv b \in C_{\ell}$. There are a set $A \subseteq \mathscr{U}$ and sequence $\bar{C}=\left\langle C_{\ell}: \ell<n(R)-2\right\rangle$ with $C_{\ell} \subseteq \mathscr{U}$ such that

(A) $|A| \leq n(R) \times n(R) \times \lambda_{1}(R)$

(B) if $\bar{b} \cong_{\emptyset} \bar{c}$ and $b_{i} E_{A, \bar{C}} c_{i}$ for all $i<\ell g(\bar{b})$ then $R(\bar{b}) \equiv R(\bar{c})$

(C) $E_{A}$ has at most $|A|+\lambda_{1}(R)$ classes

$(D)$ each $C_{\ell}$ has at most $\lambda_{1}(R)$ elements.

Proof. We try by induction on $i$ to choose $\left\langle A_{\ell}^{i}: \ell<n(R)\right\rangle$ such that

(i) $A_{\ell}^{i} \subseteq \mathscr{U}$

(ii) $\ell<k<n(R) \Rightarrow A_{\ell}^{i} \cap A_{k}^{i}=\emptyset$

(iii) $\left|A_{\ell}^{i}\right| \leq i$

(iv) $\sum_{k<n(R)}\left|\left\{\operatorname{tp}_{\mathrm{bs}}\left(b, \bigcup_{\ell \neq k} A_{\ell}^{i}\right): b \in A_{k}^{i}\right\}\right|$ is at least $i$

(v) $j<i \Rightarrow A_{\ell}^{j} \subseteq A_{\ell}^{i}$.

Now for $i=0$ let $A_{\ell}^{i}=\emptyset$.

We necessarily are stuck for some $i=i(*) \leq \lambda_{1}(R) \times n(R)$; i.e. $A_{\ell}^{j}$ are defined for $j \leq i(*)$ but we cannot choose $\left\langle A_{\ell}^{i(*)+1}: \ell<n(R)\right\rangle$, otherwise by clause (iv) for some $k$ the set $\left\{\operatorname{tp}_{\mathrm{bs}}\left(b, \bigcup_{\ell \neq k} A_{\ell}^{i}\right): b \in A_{k}^{i}\right\}$ has at least $i / n(R)$ elements which is (by the assumption toward contradiction) $>\lambda_{1}(R)$, but now $A=\bigcup_{\ell \neq k} A_{\ell}^{i}$ contradicts the definition of $\lambda_{1}(R)$ as $A_{k}^{i} \cap A=\emptyset$ by clause (ii). Let $A=\bigcup_{\ell<n(R)} A_{\ell}^{i(*)}$. For $\ell<n(R)-2$, choose $C_{\ell}$ as a set of representatives for $\left\{a / E_{A}: a / E_{A}\right.$ has $\leq n(R)$ but at least $2+\ell$ elements $\}$, such that $C_{\ell}$ is disjoint to $\bigcup_{m<\ell} C_{m}$ and we shall show that $A, \bar{C}$ is as required. Now clause $(A)$ holds by clause (iii) and the choice of $A$ (and the bound above on $i(*)$ ). Toward proving clause (B) assume $\bar{b} \cong_{\emptyset} \bar{c}$ and $b_{\ell} E_{A, \bar{C}} c_{\ell}$ for $\ell<\ell g(\bar{b})$. Without loss of generality $\bar{b}$ has no repetitions. Note if $b_{\ell} \neq c_{\ell}$ then 
$b_{\ell}, c_{\ell} \notin A\left(\right.$ as $\left.\bar{b} \approx_{A} c\right)$ and $b_{\ell} E_{A} c_{\ell}$ (see definition of $E_{A, \bar{C}}$ ), but by the choice of the $C_{\ell}$ 's, $b_{\ell} / E_{A}=c_{\ell} / E_{A}$ has $>n(R)$ elements, so there is $d \in b_{\ell} / E_{A} \backslash\left\{b_{k}: k<\ell g(\bar{b})\right\}$. Hence by transitivity of all the relevant conditions without loss of generality for some $k(*)<\ell g(\bar{b})$ we have $b_{k(*)} \neq c_{k(*)} \& \bigwedge_{m \neq k(*)} b_{m}=c_{m}$ hence $b_{k(*)}, c_{k(*)} \notin A$.

For some $t<n(R)$ we have $\left\{b_{m}: m<\ell g(\bar{b}), m \neq k(*)\right\}$ is disjoint to $A_{t}^{i(*)}$. We can find a function $\sigma$ from $\{0, \ldots, \ell g(\bar{b})-1\}$ to $\{0, \ldots, n(R)-1\}$ such that $\sigma(k)=t \equiv k=k(*), b_{m} \in A_{\ell}^{i(*)} \Rightarrow \sigma(m)=\ell$ and $\ell_{1} \neq \ell_{2} \quad \& \quad b_{\ell_{1}} \notin A \quad \& \quad b_{\ell_{2}} \notin$ $A \Rightarrow \sigma\left(\ell_{1}\right) \neq \sigma\left(\ell_{2}\right)$. For $s \in\{1,2\}$ and $r<n(R)$ let $B_{r}^{s}=A_{r}^{i(*)} \cup\left\{b_{\ell}: \sigma(\ell)=t\right\}$ if $r \neq k(*)$ and $B_{r}^{1}=A_{r}^{i(*)} \cup\left\{b_{k(*)}\right\}, B_{r}^{2}=A_{r}^{i(*)} \cup\left\{c_{k(*)}\right\}$ if $r=k(*)$. For each $s \in\{1,2\}$ we ask, choosing $\left\langle A_{r}^{i(*)+1}: r<n(R)\right\rangle$ as $\left\langle B_{r}^{s}: r<n(R)\right\rangle$ which of the demands hold. Now $B_{r}^{s}$ extends $A_{r}^{i(*)}$ (so clause (v) holds), is a subset of $\mathscr{U}$ with $\leq$ $\left|A_{r}^{i(*)}\right|+1 \leq i(*)+1$ element (by the choice of $\sigma$ ), so clauses (i) + (iii) holds and $r_{1} \neq$ $r_{2} \Rightarrow B_{r_{1}}^{s} \cap B_{r_{2}}^{s}=\emptyset$ (again look at the choice of $\sigma$ ) so clause (ii) holds. So necessarily clause (iv) fails. For $r<n(R)$ let $E_{r}$ be the following equivalence relation on $A_{r}^{i(*)}: a^{\prime} E_{r} a^{\prime \prime}$ iff $a^{\prime}, a^{\prime \prime} \in A_{r}^{i(*)}$ and $\operatorname{tp}_{\mathrm{bs}}\left(a^{\prime}, \bigcup_{m \neq r} A_{m}^{i(*)}, R\right)=\operatorname{tp}_{\mathrm{bs}}\left(a^{\prime \prime}, \bigcup_{m \neq r} A_{m}^{i(*)}, R\right)$. For $s \in\{1,2\}, k<n(R)$ let $E_{r}^{s}$ be the following equivalence relation on $B_{r}^{s}: a^{\prime} E_{r} a^{\prime \prime}$ iff $a^{\prime}, a^{\prime \prime} \in B_{r}^{s}$ and $\operatorname{tp}_{\mathrm{bs}}\left(a^{\prime}, \bigcup_{m \neq r} B_{m}^{s}, R\right)=\operatorname{tp} \mathrm{bs}\left(a^{\prime \prime}, \bigcup_{m \neq r} B_{m}^{s}, R\right)$.

Now by the definition of $E_{r}$ clearly

$$
\left|\left\{\operatorname{tp}_{\mathrm{bs}}\left(a, \bigcup_{m \neq r} A_{m}^{i(*)}, R\right): a \in A_{r}^{i(*)}\right\}\right|=\left|A_{r}^{i(*)} / E_{r}\right|
$$

hence as $\left\langle A_{r}^{i(*)}: r<n(R)\right\rangle$ satisfies $(i)-(i v)$ we know that

$$
(*)_{1} \quad i(*) \leq \sum_{r<n(R)}\left|A_{r}^{i(*)} / E_{k}\right|
$$

Also

$$
\left|\left\{\operatorname{tp}_{\mathrm{bs}}\left(a, \bigcup_{m \neq r} B_{m}^{s}, R\right): a \in B_{r}^{s}\right\}\right|=\left|B_{r}^{s} / E_{r}^{s}\right|
$$

hence as $\left\langle B_{r}^{s}: k<n(R)\right\rangle$ fail condition (iv) (see above) we have

$$
(*)_{2} \quad i(*)+1>\sum_{r<n(R)}\left|B_{r}^{s} / E_{r}\right| .
$$

Now for each $r<n(R)$, clearly $E_{r}^{s} \uparrow A_{r}^{i(*)}$ is an equivalence relation refining $E_{r}$, hence

$$
(*)_{3}\left|A_{r}^{i(*)} / E_{r}\right| \leq\left|A_{r}^{i(*)} / E_{r}^{s}\right| \leq\left|B_{r}^{s} / E_{r}^{s}\right| .
$$

The three together gives

$$
(*)_{4}\left|A_{r}^{i(*)} / E_{r}\right|=\left|A_{r}^{i(*)} / E_{r}^{s}\right|=\left|B_{r}^{s} / E_{r}^{s}\right|
$$


hence

$(*)_{5} \quad E_{r}=E_{r}^{s} \uparrow A_{r}^{i(*)}$

$(*)_{6}$ if $d \in B_{r}^{s} \backslash A_{r}^{i(*)}$ then for some $d^{\prime} \in A_{r}^{i(*)}$ we have $d E_{k}^{s} d^{\prime}$.

Apply $(*)_{6}$ to $r=t$ choosing $d_{s}=b_{k(*)}$ if $s=1$ and choosing $d_{s}=c_{k(*)}$ if $s=2$ so $d_{s} \in B_{t}^{s} \backslash A_{t}^{i(*)}$ hence there is $d_{s}^{\prime} \in A_{t}^{i(*)}$ such that $d_{s} E_{t}^{s} d_{s}^{\prime}$ so tp $\operatorname{tp}_{\mathrm{bs}}\left(d_{s}, \bigcup_{m \neq t} B_{m}^{s}, R\right)=$ $\operatorname{tp}_{\mathrm{bs}}\left(d_{s}^{\prime}, \bigcup_{m r \neq t} B_{m}^{s}, R\right)$ hence $\operatorname{tp}_{\mathrm{bs}}\left(d_{s}, \bigcup_{m \neq t} A_{m}^{i(*)}, R\right)=\operatorname{tp}_{\mathrm{bs}}\left(d_{s}^{\prime}, \bigcup_{m \neq t} A_{m}^{i(*)}, R\right)$.

But $\left.\operatorname{tp}_{\mathrm{bs}}\left(d_{1}, \bigcup_{m \neq t} A_{m}^{i(*)}, R\right)=\operatorname{tp}_{\mathrm{bs}}\left(b_{k(*)}, \bigcup_{m \neq t} A_{m}^{i(*)}, R\right)=\operatorname{tp} \operatorname{tbs}_{\left(c_{k(*)}\right.}, \bigcup_{m \neq t} A_{m}^{i(*)}, R\right)=$ $\operatorname{tp} \mathrm{bs}\left(d_{2}, \bigcup_{m \neq t} A_{t}^{i(*)}, R\right)$ (second equality as $b_{k(*)} E_{A} c_{k(*)}$ by the choice of $\bar{b}, \bar{c}$ ).

So together with the previous sentences tp $\operatorname{bs}\left(d_{1}^{\prime}, \bigcup_{m \neq t} A_{m}^{i(*)}, R\right)=\operatorname{tp}_{\mathrm{bs}}\left(d_{2}^{\prime}, \bigcup_{m \neq t} A_{m}^{i(*)}, R\right)$ that is $d_{1}^{\prime} E_{t} d_{2}^{\prime}$ (recall $d_{1}^{\prime}, d_{2}^{\prime} \in A_{t}^{i(*)}$ ). So by $(*)_{5}$ we have $d_{1}^{\prime} E_{t}^{s} d_{2}^{\prime}$ for $s=1,2$. Clearly $m<n(R) \quad \& \quad m \neq t \Rightarrow B_{m}^{1}=B_{m}^{2}$ hence $\bigcup_{m \neq t} B_{m}^{1}=\bigcup_{m \neq t} B_{m}^{2}$ and let $E_{t}^{*}$ be the following equivalence relation on $\mathscr{U}: a^{\prime} E_{t}^{*} a^{\prime \prime}$ iff $\operatorname{tp}_{\mathrm{bs}}\left(a^{\prime}, \bigcup_{m \neq t} B_{m}^{s}, R\right)=$ $\operatorname{tp}_{\mathrm{bs}}\left(a^{\prime \prime}, \bigcup_{m \neq t} B_{m}^{s}, R\right)$. Clearly $E_{t}^{s}=E_{s}^{*} \uparrow B_{t}^{s}$, hence $d_{1} E_{t}^{*} d_{1}^{\prime}$ (by the choice of $d_{1}^{\prime}$ ), $d_{1}^{\prime} E_{t}^{*} d_{2}^{\prime}$ (see the previous sentences) and $d_{2}^{\prime} E_{t}^{*} d_{2}$ (by the choice of $d_{2}^{\prime}$ ). Together as $d_{1}=b_{k(*)}, d_{2}=c_{k(*)}$ we have $b_{k(*)} E_{t}^{*} c_{k(*)}$; but $\left\{c_{\ell}: \ell \neq k(*)\right\}=\left\{b_{\ell}: \ell \neq k(*)\right\} \subseteq$ $\bigcup_{m \neq t} B_{m}^{s}$ (by the choice of $\sigma$ ) so tp $\operatorname{tbs}\left(b_{k(*)},\left\{c_{\ell}: \ell \neq k(*)\right\}, R\right)=\operatorname{tp}_{\mathrm{bs}}\left(c_{k(*)},\left\{c_{\ell}: \ell \neq\right.\right.$ $k(*)\}, R)$ a contradiction to the choice of $\bar{b}, \bar{c}$.

So $A=\bigcup_{r<n(R)} A_{r}^{i(*)}$ satisfies clause (B) of 4.4. Note that $E_{A}$ has $\leq|A|+\lambda_{1}(R)$ equivalence classes by the definition of $\lambda_{1}(R)$, so $A$ satisfies clause (C), and $\bar{C}$ satisfies clause (D) so is really as required.

4.5 Conclusion. Letting $\lambda_{\ell}=\lambda_{\ell}(R)$ we have $\exists_{R}$ is bi-interpretable with $\left\{Q_{\lambda_{0}}^{\text {mon }}, Q_{\lambda_{1}}^{1-1}, \exists_{R_{1}}, \exists_{E}\right\}$, where $\left|\operatorname{Dom}\left(R_{1}\right)\right| \leq n(R)^{2} \lambda_{1}(R)$ and $E$ is an equivalence relation on $\mathscr{U}$. This is done uniformly (i.e. the formulas depend on $n(R)$ only).

Remark. Note that $Q_{\lambda_{0}}^{\text {mon }}$ can be omitted being swallowed by $\exists_{E}$.

Proof. We've shown $Q_{\lambda_{1}(R)}^{1-1} \leq_{\text {int }} \exists_{R}$ (see 4.3). Let $A, C$ be as in the lemma 4.4, choose $A_{1}$ such that $A^{1} \cap A=\emptyset,\left|A^{1}\right| \leq n(R)^{2} \lambda_{1}(R)$ and $A \cup A^{1}$ includes $\geq \operatorname{Min}\left\{n(R),\left|a / E_{A}\right|\right\}$ elements of each $E_{A}$ equivalence class $a / E_{A}$. Lastly let $R_{1}=R \uparrow\left(A \cup A^{1}\right)$.

Now by the choice of $A$ and $\bar{C}$ clearly

$$
R\left(x_{1}, \ldots, s_{n(R)}\right) \text { iff }
$$




$$
\begin{gathered}
\left(\exists y_{1}\right) \cdots\left(\exists y_{n(R)}\right)\left(\bigwedge_{1 \leq i \leq n(R)} x_{i} E_{A, \bar{C}} y_{i} \& \bigwedge_{i, j=1, \ldots, n(R)} x_{1}=x_{j} \equiv y_{i}=y_{j}\right. \\
\left.\& R_{1}(\bar{y})\right) .
\end{gathered}
$$

So $\exists_{R} \leq_{\text {int }}\left\{Q_{\lambda_{1}}^{1-1}, \exists_{R_{1}}, \exists_{E_{A, \bar{C}}}\right\}$. Now $\exists_{R_{1}} \leq_{\text {int }}\left\{\exists_{R}, Q_{\lambda_{1}}^{\text {mon }}\right\}$ by the definition of $R_{1}, \exists_{E_{A, \bar{C}}} \leq_{\text {int }}\left\{\exists_{R}, Q_{\lambda_{1}}^{\text {mon }}\right\}$ directly and $Q_{\lambda_{1}}^{1-1} \leq \exists_{R}$ by 4.3 and $Q_{\lambda_{1}}^{\text {mon }} \leq Q_{\lambda_{1}}^{1-1}$. So $\left\{Q_{\lambda_{1}}^{1-1}, Q_{\lambda_{0}}^{\text {mon }}, \exists_{R_{1}}, \exists_{E_{A}}\right\} \leq_{\text {int }}\left\{\exists_{R}, Q^{\text {mon }} \lambda_{0}\right\}$ and we finish. $\quad \square_{\mathbf{4 . 5}}$ $\rightarrow \quad$ scite $\{3.5\}$ undefined

4.6 Remark. Note the $Q_{\left|\operatorname{Dom}\left(R_{1}\right)\right|}^{1-1}$ is uniformly interpretable (for fixed $n(R)$ ) in $Q_{\lambda_{1}}^{1-1}$ including the case $\lambda_{1}$ is finite, so $\mathbf{4 . 5}$ holds for it too.

$\rightarrow \quad$ scite $\{3.5\}$ undefined

4.7 Claim. If $|\mathscr{U}|>\lambda_{1} \geq \lambda^{k}, R$ a k-place relation on $A \subseteq \mathscr{U}$ and $|A| \leq \lambda$ (and $\mathscr{U}$ finite) then $Q_{R} \leq \exp Q_{\lambda_{1}}^{1-1}$.

Proof. By 2.12 .

4.8 Conclusion. If $R$ is an $n(R)$-place relation on $\mathscr{U}$ and $\lambda_{1}(R)^{n(R)} \leq|\mathscr{U}|$, then for some equivalence relation $E$ we have

$$
\left\{Q_{E}^{\mathrm{eq}}, Q_{\lambda_{1}(R)}^{1-1}\right\} \leq_{\text {int }} Q_{R} \leq_{\exp }\left\{Q_{E}^{\mathrm{eq}}, Q_{\lambda_{1}(R)^{n(R)}}^{1-1}\right\} .
$$

Proof. We have by 4.3 that $Q_{\lambda_{1}(R)}^{1-1} \leq \exists_{R}$. By 4.7 for every binary relation $S$ on $\sqrt{\lambda_{1}}$ we have $\exists_{S} \leq Q_{\lambda_{1}(R)}^{1-1}$. So every relation on $\mathscr{U}^{\frac{1}{2 n(R)}}$ is interpreted in $\exists_{R}$.

4.9 Remark. So up to expressability and up to a power by $n(R)$ (and possibly increasing $\mathscr{U}$ ), we have that $\left\{Q_{E}^{\mathrm{eq}}, Q_{\lambda_{1}(R)}^{1-1}\right\}$ exhaust all the information on $Q_{R}$ (up to interpretability).

We can get the parallel result for $Q_{K}$.

4.10 Definition. $\lambda_{1}(K)=\left\{\lambda\right.$ : for every $R \in K$ we have $\left.\lambda_{1}(R)<\lambda\right\}$. Note that the maximum is taken for each $\mathscr{U}$ separately.

4.11 Conclusion. 1) $Q_{<\lambda_{1}(K)}^{1-1} \leq_{\text {int }} \exists_{K}$.

2) There are $K_{1}$ and $\mathbf{E}$, a family of equivalence relations (for each $\mathscr{U} \in \mathfrak{U}$, closed under permutations of $\mathscr{U}$ ) such that:

(a) $\exists_{K} \equiv_{\mathrm{int}}\left\{\exists_{K_{1}}, Q_{<\lambda_{1}(K)}^{1-1}, Q_{\mathbf{E}}\right\}$

(b) for any $R \in K_{1}$ we have $|\operatorname{Dom}(R)|<n(R)^{2} \times \mu(K)$ where $\mu(K)=\operatorname{Min}\{\mu$ : $R \in K \Rightarrow|\operatorname{Dom}(R)|<\mu\}$ the minimum taken for each $\mathscr{U} \in \mathfrak{U}$ separately.

Proof. Straight by uniformity. 
Assignments
1) $2.10 ; 2$ copies
2) $639 \mathrm{a}$ 


\section{REFERENCES.}

[Bl] John T. Baldwin. Definable second order quantifiers. In J. Barwise and S. Feferman, editors, Model Theoretic Logics, Perspectives in Mathematical Logic, chapter XII, pages 446-478. Springer-Verlag, New York Berlin Heidelberg Tokyo, 1985.

[BlSh 156] John T. Baldwin and Saharon Shelah. Second-order quantifiers and the complexity of theories. Notre Dame Journal of Formal Logic, 26:229303, 1985. Proceedings of the 1980/1 Jerusalem Model Theory year.

[Sh:e] Saharon Shelah. Non-structure theory, accepted. Oxford University Press.

[Sh 28] Saharon Shelah. There are just four second-order quantifiers. Israel Journal of Mathematics, 15:282-300, 1973.

[Sh 171] Saharon Shelah. Classifying of generalized quantifiers. In Around classification theory of models, volume 1182 of Lecture Notes in Mathematics, pages 1-46. Springer, Berlin, 1986.

[Sh:F334] Shelah, Saharon. On quantification with a finite universe II. 